This item was submitted to Loughborough's Research Repository by the author.

Items in Figshare are protected by copyright, with all rights reserved, unless otherwise indicated.

\title{
An interaction theory for scattering by defects in arrays
}

PLEASE CITE THE PUBLISHED VERSION

PUBLISHER

(C) Society for Industrial and Applied Mathematics

VERSION

VoR (Version of Record)

LICENCE

CC BY-NC-ND 4.0

REPOSITORY RECORD

Thompson, lan, and C.M. Linton. 2019. "An Interaction Theory for Scattering by Defects in Arrays". figshare. https://hdl.handle.net/2134/4261. 
This item was submitted to Loughborough's Institutional Repository (https://dspace.lboro.ac.uk/) by the author and is made available under the following Creative Commons Licence conditions.

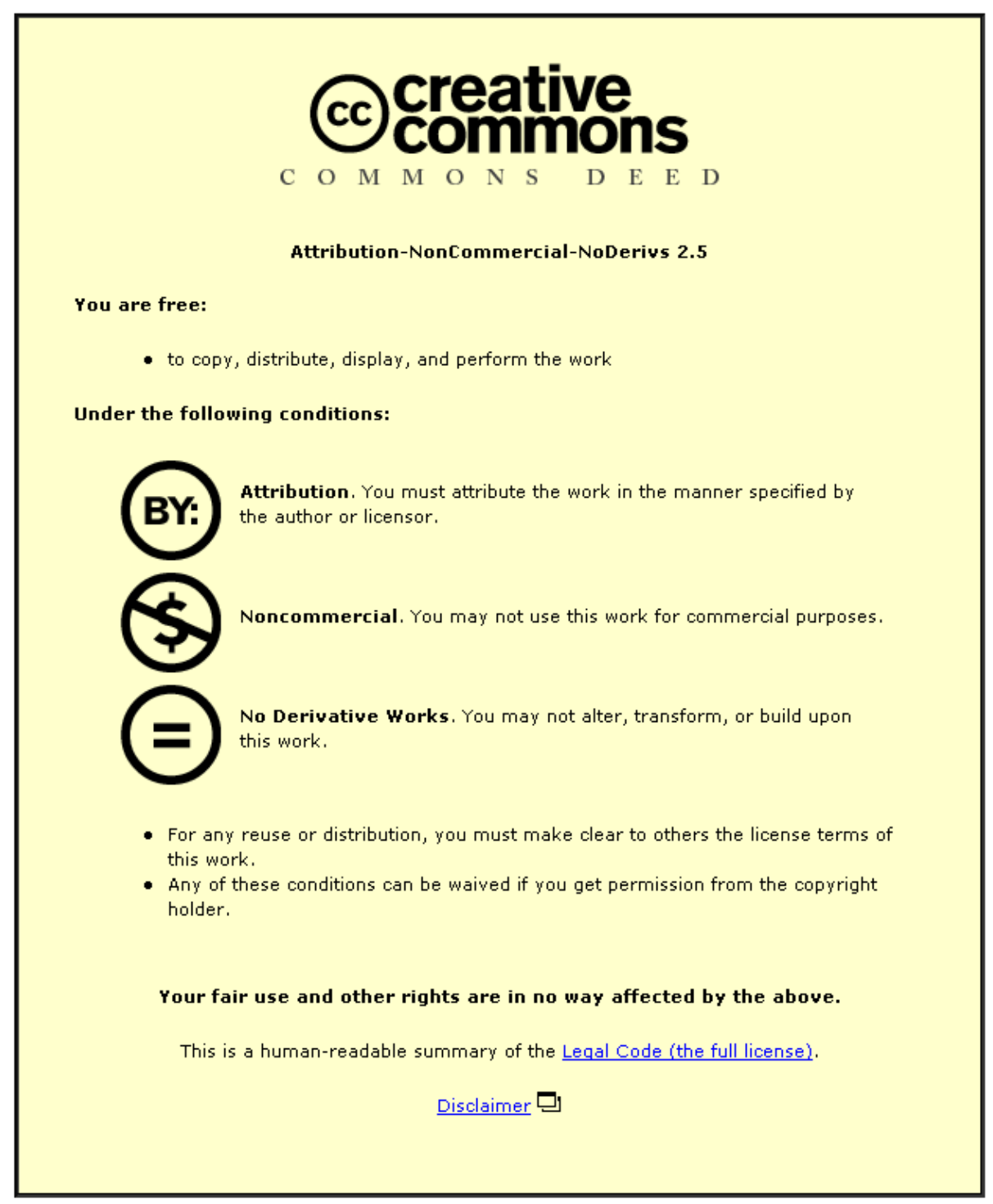

For the full text of this licence, please go to: http://creativecommons.org/licenses/by-nc-nd/2.5/ 


\title{
AN INTERACTION THEORY FOR SCATTERING BY DEFECTS IN ARRAYS*
}

\author{
I. THOMPSON $^{\dagger}$ AND C. M. LINTON ${ }^{\dagger}$
}

\begin{abstract}
Wave scattering by an array of bodies that is periodic except for a finite number of missing or irregular elements is considered. The field is decomposed into contributions from a set of canonical problems, which are solved using a modified array scanning method. The resulting interaction theory for defects is very efficient and can be used to construct the field in a large number of different situations. Numerical results are presented for several cases, and particular attention is paid to the amplitude with which surface waves are excited along the array. We also show how other approaches can be incorporated into the theory so as to increase the range of problems that can be solved.
\end{abstract}

Key words. arrays, defects, scattering, surface waves

AMS subject classifications. 78A45, 78A50, 42A99

DOI. $10.1137 / 070703144$

1. Introduction. Wave scattering by arrays of bodies is of fundamental importance in numerous engineering and physics applications. Here we are concerned with the effect of one or more defects in an infinite, periodic array. This problem is of significant current interest in several fields, including elastodynamics [19] and phononic $[7,28]$ and photonic $[1,26,5]$ crystals. The presence of defects leads to a significant increase in difficulty in determining the scattered field, because the geometry is no longer periodic. In particular, Rayleigh-Bloch (RB) surface waves (also known as array guided surface waves) are excited if the array geometry and physical parameters are such that these modes can exist. RB waves propagate without loss along an array, and are evanescent in other directions. They are known to occur in a wide variety of situations $[15,21,18,10]$. One of the key goals of this article is to develop an efficient and accurate method for the determination of the amplitude with which they are excited. The theory is presented in a form that can be directly interpreted in a number of different physical contexts. These include the acoustic case, in which the wavenumber $k$ is the ratio of the angular frequency $\omega$ to the speed of sound $c$, and the interaction of linear water waves with bottom mounted, surface penetrating cylinders, in which case $k$ is the positive solution to the dispersion relation $k \tanh k h=\omega^{2} / g$, $g$ being the acceleration due to gravity and $h$ the quiescent fluid depth. For acoustics, Dirichlet and Neumann boundary conditions are used to model sound hard and sound soft bodies, respectively, whereas Neumann conditions are appropriate for solid bodies immersed in water. The method is also applicable in the electromagnetic and elastodynamic cases, provided that the overall vector wave problem decouples into separate scalar components.

Our first step in obtaining the field scattered by a defective array is to decompose the solution into contributions arising from a set of simpler, canonical problems. This is achieved by modifying the field generated when a wave interacts with a periodic

* Received by the editors September 19, 2007; accepted for publication (in revised form) March 24, 2008; published electronically July 16, 2008.

http://www.siam.org/journals/siap/68-6/70314.html

${ }^{\dagger}$ Department of Mathematical Sciences, Loughborough University, Loughborough, Leicestershire, UK (i.thompson@lboro.ac.uk, c.m.linton@lboro.ac.uk). The first author's research was supported by EPSRC grant EP/C510941/1. 
array, so as to eliminate a finite number of elements, or replace these with bodies of different sizes, shapes, or surface compositions. The procedure is independent of the type of wavefunctions used to represent the field (i.e., cylindrical, spherical, etc.) and is therefore presented in a general form in section 2. The canonical problems are independent of the defect configuration and all aspects of the incident field, except the wavenumber; they need not be solved again if these parameters are changed. In order for the decomposition to be useful in a specific case, the relevant canonical problems must be solved accurately and efficiently. The boundary conditions on the surface of the array elements come into play at this stage, and therefore we must apply an appropriate multiple scattering theory. This requires the use of certain results concerning the periodic array, and these are readily available for problems involving cylindrical wavefunctions; a summary is given in section 3. The canonical problems for this case are then solved in section 4 using a special Fourier series. This approach is closely related to the array scanning method $[27,16]$, which is typically used in problems involving excitation by an aperiodic field, and in particular for the analysis of antenna arrays [3, 4]. The idea is to create a periodic incident field by introducing an array of phase-shifted sources, and then to integrate over a single period of the phase shift so as to eliminate all but one of the sources. The procedure used in section 4 is similar, but its effect is rather different, and we shall refer to it as the "modified array scanning method" (MASM). Instead of eliminating sources, the integration, which must be performed using quadrature, enables us to replace one member of a periodic array with a source. This is the most computationally intensive part of the technique. Nevertheless, important parameters such as RB wave amplitudes can be efficiently calculated to near machine precision. In contrast, other techniques such as the filtering approach used for a related problem in [11] have limited accuracy. Technical details regarding the method used to evaluate the relevant integrals are given in the appendix. This method is chosen for simplicity and is open to improvement.

Considered together, the decomposition into canonical problems and the MASM are similar to the "fictitious source superposition method" which was originally used for a study of photonic crystals with a single defect [26]. This was later extended in [5] to account for situations where more than one defect is present. Our formulation, which is a generalization of earlier work in [22], is rather different and automatically includes the case of multiple defects. Indeed, by first reducing to canonical problems, we obtain an "interaction theory for defects" by means of which the solutions for a wide variety of cases can be constructed at very little computational expense.

A representative sample of the numerical results that can be obtained is given in section 5. We also demonstrate how the methods in sections 2-4 can be combined with other approaches, such as infinite array subtraction [11] and the large array approximation method used in [24], to widen the class of problems that can be considered.

2. General theory. In this section we will show how the problem of wave scattering by a defective array can be reduced to a set of simpler, canonical problems. This is achieved using a procedure that is independent of the shape of the scatterers and the boundary conditions that are to be applied on their surfaces. We therefore present the theory from a general perspective, although for clarity we deal with the case of a one-dimensional array in the two-dimensional setting. The extensions to higher array dimensions and to three dimensions in space is straightforward, requiring only that scalar indices are replaced by appropriate multi-indices.

Thus, consider an array of scatterers which is periodic, except for a finite number 
of missing, or possibly irregular, elements. The elements are labeled by an index $p \in \mathbb{Z}$, and the defects correspond to those values for which $p$ is a member of the finite defect set $\mathcal{D}$. If $p \notin \mathcal{D}$, we shall say that scatterer $p$ is regular. A (one-dimensional) lattice of points $\mathbf{r}_{p}$ is defined so that $\mathbf{r}=\mathbf{r}_{p}$ lies inside scatterer $p$ if this body is present in the array. The field in the vicinity of each scatterer is then expanded about the point $\mathbf{r}=\mathbf{r}_{p}$ as a sum of incoming and outgoing wavefunctions. The former are regular for all $\mathbf{r}$, whereas the latter are singular at $\mathbf{r}=\mathbf{r}_{p}$ and regular elsewhere. The choice of $\mathbf{r}_{p}$ is of course not unique.

In the region exterior to the scatterers, all wavefields $\phi$ must satisfy the Helmholtz equation

$$
\left(\nabla^{2}+k^{2}\right) \phi=0 .
$$

The array is excited by the incident wave $\phi^{\mathrm{i}}$, and the total field is obtained by adding the scattered response. Hence,

$$
\phi^{\mathrm{t}}=\phi^{\mathrm{i}}+\phi^{\mathrm{s}},
$$

where $\phi^{\mathrm{s}}$ can be expanded in the form

$$
\phi^{\mathrm{s}}(\mathbf{r} ; \mathcal{D})=\sum_{m} \sum_{p} A_{m}^{p}(\mathcal{D}) \mathcal{H}_{m}^{p}(\mathbf{r}) .
$$

Here, the notation $\mathcal{H}_{m}^{p}$ represents an outgoing wavefunction of order $m$ that is singular at $\mathbf{r}=\mathbf{r}_{p}$ and regular elsewhere. Where no limits are placed on an index it is to be understood that this ranges over all possible values. The radiation condition stipulates that $\phi^{\mathrm{s}}$ cannot include any contributions that are incoming from the far field, or that increase in magnitude as the observer moves toward infinity. Initially, we consider defects that consist of missing scatterers, in which case we must have

$$
A_{m}^{p}(\mathcal{D})=0, \quad p \in \mathcal{D},
$$

so that there are no singularities in the field. Later we will show how the theory can be modified to account for irregular scatterers, which is slightly more difficult.

The pivotal idea behind our procedure is to modify $\phi^{\mathrm{s}}(\mathbf{r}, \emptyset)$ (i.e., the scattered field that occurs when there is no defect) by cancelling the singularities at $\mathbf{r}=\mathbf{r}_{p}$ for each $p \in \mathcal{D}$. The resulting wavefield does not include any radiation from the scatterers for which $p \in \mathcal{D}$, and no longer satisfies the boundary conditions on their surface. In this way, the influence of these array elements is eliminated. The boundary conditions on the surface of the regular scatterers are still satisfied, as is the radiation condition.

At a later stage, it is necessary to apply a multiple scattering theory in order to satisfy the boundary conditions on the scatterer surfaces. This requires that, in some region containing the surface of scatterer $p$, the total field can be represented in the form

$$
\phi^{\mathrm{t}}(\mathbf{r} ; \mathcal{D})=\phi_{p}^{\mathrm{i}}(\mathbf{r} ; \mathcal{D})+\phi_{p}^{\mathrm{r}}(\mathbf{r} ; \mathcal{D})
$$

where

$$
\phi_{p}^{\mathrm{i}}(\mathbf{r} ; \mathcal{D})=\sum_{m} I_{m}^{p}(\mathcal{D}) \mathcal{J}_{m}^{p}(\mathbf{r})
$$

Copyright (C) by SIAM. Unauthorized reproduction of this article is prohibited. 
and

$$
\phi_{p}^{\mathrm{r}}(\mathbf{r} ; \mathcal{D})=\sum_{m} A_{m}^{p}(\mathcal{D}) \mathcal{H}_{m}^{p}(\mathbf{r})
$$

Here, $\mathcal{J}_{m}^{p}$ represents a regular wavefunction of order $m$ and $\phi_{p}^{\mathrm{i}}$ is the total field incoming toward the point $\mathbf{r}_{p}$. It consists of the incident wave and the radiation from all of the other scatterers. The second term on the right-hand side of (2.5) represents the field outgoing from scatterer $p$. The relationships between the expansions (2.3) and (2.5)-(2.7) can be found in [14, Chapters 2 and 3] for wavefunctions in a number of separable geometries. The crucial point here is the nature of the regions where the series appearing in (2.6) and (2.7) converge and therefore represent valid solutions to the Helmholtz equation. The expansion of the incoming field (2.6) is valid inside a simply connected region that contains the point $\mathbf{r}_{p}$. In fact, if we are to apply a multiple scattering theory based on the expansions (2.5)-(2.7), this region must contain the whole of scatterer $p$. Thus, the field incoming toward a particular body can be extended to the entire region inside that body, and there it continues to represent a valid solution to the Helmholtz equation. The same cannot be said for the field radiating from a particular body (equation (2.7)) because $\mathcal{H}_{m}^{p}(\mathbf{r})$ is singular at the point $\mathbf{r}=\mathbf{r}_{p}$. Note that the use of (2.5)-(2.7) to represent the field at the surface of the scatterers imposes a geometrical restriction. For cylindrical and spherical wavefunctions, the maximum distance from $\mathbf{r}_{p}$ to the surface of scatterer $p$ must be less than $\left|\mathbf{r}_{p}-\mathbf{r}_{p \pm 1}\right|$ [14, sections 2.5, 3.12].

As a starting point, for the case where $\mathcal{D}=\emptyset$, we have

$$
\phi^{\mathrm{s}}(\mathbf{r} ; \emptyset)=\sum_{m} \sum_{p} A_{m}^{p}(\emptyset) \mathcal{H}_{m}^{p}(\mathbf{r}),
$$

and we will assume that the coefficients $A_{m}^{p}(\emptyset)$ are known, since this is a periodic geometry, and so the solution can be obtained relatively easily. Now, construct the field $\phi^{\mathrm{s}}(\mathbf{r} ; \mathcal{D})$ by writing

$$
\phi^{\mathrm{s}}(\mathbf{r} ; \mathcal{D})=\phi^{\mathrm{s}}(\mathbf{r} ; \emptyset)+\psi(\mathbf{r} ; \mathcal{D}),
$$

and observe that $\psi(\mathbf{r} ; \mathcal{D})$ must satisfy the boundary conditions on the regular scatterers because $\phi^{\mathrm{s}}(\mathbf{r} ; \mathcal{D})$ and $\phi^{\mathrm{s}}(\mathbf{r} ; \emptyset)$ do so independently. From (2.3), (2.4), and (2.8) we have the explicit representation

$$
\psi(\mathbf{r} ; \mathcal{D})=\sum_{m} \sum_{p \notin \mathcal{D}}\left[A_{m}^{p}(\mathcal{D})-A_{m}^{p}(\emptyset)\right] \mathcal{H}_{m}^{p}(\mathbf{r})-\sum_{m} \sum_{p \in \mathcal{D}} A_{m}^{p}(\emptyset) \mathcal{H}_{m}^{p}(\mathbf{r}) .
$$

By considering the last term on the right-hand side (which is known) as an incident field, and the other terms as the associated scattered response, it is now seen that $\psi(\mathbf{r} ; \mathcal{D})$ is the total field that occurs when an array with scatterers absent for $p \in \mathcal{D}$ is excited by a distribution of sources located at the points $\mathbf{r}=\mathbf{r}_{p}, p \in \mathcal{D}$. We shall refer to $\mathcal{H}_{m}^{p}(\mathbf{r})$ as the source of order $m$ with unit amplitude, located at the point $\mathbf{r}=\mathbf{r}_{p}$.

Rather than solve for $\psi(\mathbf{r} ; \mathcal{D})$ directly, we can reduce the problem to a set of simpler, canonical problems by considering each source term in (2.10) separately. Thus, introduce the potential $\psi_{n}^{q}(\mathbf{r})$, which represents the total field that occurs when a periodic array has a single element (labeled by $q$ ) removed and replaced by a unit 
source of order $n$. Crucially, if $q \in \mathcal{D}$, then $\psi_{n}^{q}(\mathbf{r})$ satisfies the boundary conditions on the surface of all the regular scatterers. Now $\psi(\mathbf{r} ; \mathcal{D})$ clearly consists entirely of waves that are outgoing from the array, and therefore we can expand it into the form

$$
\psi_{n}^{q}(\mathbf{r})=\mathcal{H}_{n}^{q}(\mathbf{r})+\sum_{m} \sum_{p \neq q} C_{m, n}^{p, q} \mathcal{H}_{m}^{p}(\mathbf{r})
$$

Here, we have introduced the convention that the indices to the right of the comma describe the source, in this case referring to order $n$ and position $q$. It is convenient to simplify such expressions by defining

$$
C_{m, n}^{q, q}=\delta_{m n}
$$

so that the first term on the right-hand side can be taken inside the series. To avoid any possible misinterpretation, we emphasize that (2.11) does not represent a homogeneous solution to the periodic (i.e., defect-free) array problem because the appropriate boundary condition on the surface of scatterer $q$ is not satisfied.

Next, we represent $\psi(\mathbf{r} ; \mathcal{D})$ as a linear combination of the potentials $\psi_{n}^{q}(\mathbf{r}), q \in \mathcal{D}$; thus

$$
\psi(\mathbf{r} ; \mathcal{D})=\sum_{n} \sum_{q \in \mathcal{D}} a_{n}^{q} \psi_{n}^{q}(\mathbf{r})
$$

If we substitute from (2.11) into (2.13) and rearrange the summations, we obtain

$$
\psi(\mathbf{r} ; \mathcal{D})=\sum_{m} \sum_{p \in \mathcal{D}} a_{m}^{p} \mathcal{H}_{m}^{p}(\mathbf{r})+\sum_{m} \sum_{p} \sum_{n} \sum_{\substack{q \in \mathcal{D} \\ q \neq p}} a_{n}^{q} C_{m, n}^{p, q} \mathcal{H}_{m}^{p}(\mathbf{r}) .
$$

Comparing this with (2.10), we find that

$$
a_{m}^{p}+\sum_{n} \sum_{\substack{q \in \mathcal{D} \\ q \neq p}} a_{n}^{q} C_{m, n}^{p, q}=-A_{m}^{p}(\emptyset), \quad p \in \mathcal{D},
$$

which is a linear system of equations for the coefficients $a_{m}^{p}$, and

$$
\sum_{n} \sum_{q \in \mathcal{D}} a_{n}^{q} C_{m, n}^{p, q}=A_{m}^{p}(\mathcal{D})-A_{m}^{p}(\emptyset), \quad p \notin \mathcal{D},
$$

which then serves to determine the unknowns $A_{m}^{p}(\mathcal{D})$. Equation $(2.15)$ is an "interaction theory for defects," which is similar in nature to the standard interaction theories for multiple bodies. If only a single scatterer is absent from the array, we retrieve $a_{m}^{p}=-A_{m}^{p}(\emptyset)$ so as to cancel the radiation emanating from $\mathbf{r}=\mathbf{r}_{p}$, as we should expect. A useful simplification now occurs if the array consists of periodic repetitions of a single body. In this case the potentials $\psi_{m}^{p}$ are identical up to a spatial shift, and we need only determine $\psi_{m}^{0}$. In terms of the coefficients $C_{m, n}^{p, q}$, we have

$$
C_{m, n}^{p, q}=C_{m, n}^{p-q, 0}
$$

and so there is a single canonical problem to solve for each value of $m$.

Finally, consider defects consisting of scatterers that are in some way different from the other elements of the array. In this case, the method operates by replacing 
members of the periodic array for which $p \in \mathcal{D}$ with irregular bodies. In contrast to the case of absent scatterers, $A_{m}^{p}(\mathcal{D})$ is generally nonzero for $p \in \mathcal{D}$. The singularities at $\mathbf{r}=\mathbf{r}_{p}$ are no longer cancelled; instead they are adjusted so that for $p \in \mathcal{D}$, the expansion (2.7) represents a solution to the Helmholtz equation in the region exterior to the new element. Consequently, the point $\mathbf{r}=\mathbf{r}_{p}$ must lie inside scatterer $p$ for $p \in \mathcal{D}$ (as it does for $p \notin \mathcal{D}$ ). The field $\psi(\mathbf{r} ; \mathcal{D})$ can still be constructed from a linear combination of the solutions to the same canonical problems, but in place of (2.15), we now have

$$
a_{m}^{p}+\sum_{n} \sum_{\substack{q \in \mathcal{D} \\ q \neq p}} a_{n}^{q} C_{m, n}^{p, q}=A_{m}^{p}(\mathcal{D})-A_{m}^{p}(\emptyset), \quad p \in \mathcal{D} .
$$

Equation (2.16) is unaffected. The presence of the additional unknowns $A_{m}^{p}(\mathcal{D})$ on the right-hand side of (2.18) is countered by the need to apply a boundary condition on the surface of the irregular scatterers, and in section 5 we shall see how this works in practice. While it is evident that replacing scatterers is more complicated than eliminating them, the increase in difficulty is marginal. Essentially this is because the extra requirement is to determine the field incoming toward $\mathbf{r}=\mathbf{r}_{p}$ for $p \in \mathcal{D}$, but this is no more difficult than determining the field incoming toward a regular scatterer, which is always necessary.

A major advantage of this method over a more direct approach is as follows. Had we simply applied an interaction theory to the defective array problem, we would be faced with the inversion of a linear system of equations containing infinite sums over the spatial indices. These have a very slow rate of convergence and present serious difficulties in obtaining accurate results, even with the aid of modern computing power. In contrast, (2.15), (2.16), and (2.18) contain only finite spatial sums. The infinite order summation is of less concern, particularly at low frequencies, because as $|m|$ is increased the coefficients $A_{m}^{p}$ converge rapidly to zero. Even in cases where the scatterers are almost in contact, the convergence of the order sum is much more rapid than that of the spatial sum; the former can be truncated at a relatively small value of $|m|$. Of course, it remains to solve the canonical problems, and these involve infinite linear systems containing spatial sums. However, these possess symmetries that are not present in the overall problem, and as mentioned earlier, solutions to one set of canonical problems can be used to construct the field for a number of different cases. Thus, the decomposition described above is useful even in problems where the MASM cannot be used effectively.

3. Array problems involving cylindrical wavefunctions. In order to solve the canonical problems that arise in the interaction theory for defects, we must deal with the boundary conditions on the scatterer surfaces. It is therefore necessary to present subsequent material for a specific geometry, and since the theory of linear arrays is well established for the case of cylindrical wavefunctions, this is a natural choice. Here, we collect some results from pre-existing literature in this area that will be needed later. It should be noted that the essential principles upon which the method depends remain unchanged if wavefunctions from another separable geometry are used. We will assume that the scatterers themselves are circular so as to present the theory in the simplest possible form; however we will indicate how scatterers of a different shape can be considered through the incorporation of transfer matrices.

Let all lengths be scaled on the distance between the centers of consecutive lattice points, with these located at $\mathbf{r}_{p}=(p, 0)$ in the $(x, y)$ plane. According to the chosen 
scaling, the radius $a$ of the regular scatterers must satisfy the inequality $a \leq 0.5$. The expansion (2.3) now takes the form

$$
\phi^{\mathrm{s}}(\mathbf{r} ; \mathcal{D})=\sum_{m} \sum_{p} A_{m}^{p}(\mathcal{D}) H_{m}^{(1)}\left(k r_{p}\right) \mathrm{e}^{\mathrm{i} m \theta_{p}}
$$

where $\left(r_{p}, \theta_{p}\right)$ is a set of polar coordinates with its origin at the center of scatterer $p$ (see Figure 3.1), and $H_{m}^{(1)}(\cdot)$ denotes a Hankel function of the first kind with order $m$. This choice of outgoing wavefunction (rather than $H_{m}^{(2)}(\cdot)$ ) corresponds to an implicit time-harmonic factor $\mathrm{e}^{-\mathrm{i} \omega t}$. We also have a decomposition of the form (2.5)-(2.7), with

$$
\phi_{p}^{\mathrm{i}}\left(r_{p}, \theta_{p} ; \mathcal{D}\right)=\sum_{m} I_{m}^{p}(\mathcal{D}) J_{m}\left(k r_{p}\right) \mathrm{e}^{\mathrm{i} m \theta_{p}}
$$

and

$$
\phi_{p}^{\mathrm{r}}\left(r_{p}, \theta_{p} ; \mathcal{D}\right)=\sum_{m} A_{m}^{p}(\mathcal{D}) H_{m}^{(1)}\left(k r_{p}\right) \mathrm{e}^{\mathrm{i} m \theta_{p}},
$$

where $J_{m}(\cdot)$ is the Bessel function of order $m$. As before, $\phi_{p}^{\mathrm{i}}(\mathbf{r} ; \mathcal{D})$ represents the total field incoming toward scatterer $p$, and this consists of the incident wave and the radiation from all of the other scatterers. The expansion (3.2) is a valid representation for $\phi_{p}^{\mathrm{i}}(\mathbf{r} ; \mathcal{D})$, provided that $r_{p}<1$. In general, a transfer matrix appropriate to the geometry of the scatterers relates the coefficients $I_{m}^{p}(\mathcal{D})$ and $A_{m}^{p}(\mathcal{D})$, but for circular scatterers, orthogonality leads to a matrix that is diagonal. Consequently, we can write

$$
A_{m}^{p}(\mathcal{D})+Z_{m} I_{m}^{p}(\mathcal{D})=0
$$

where $Z_{m}$ is a scattering coefficient which is given by

$$
Z_{m}=J_{m}(k a) / H_{m}^{(1)}(k a)
$$

for Dirichlet boundary conditions, or

$$
Z_{m}=J_{m}^{\prime}(k a) / H_{m}^{(1) \prime}(k a)
$$

for Neumann conditions. Other expressions for $Z_{m}$ can be used to model different situations, such as impedance boundary conditions.

Scattering problems of this type can be separated into components that are symmetric and antisymmetric about $y=0$ by decomposing the incident field $\phi^{\mathrm{i}}$ into an even (subscript "+") and an odd (subscript "-") function of $y$; thus

$$
\phi_{ \pm}^{\mathrm{i}}(x, y)=\frac{1}{2}\left[\phi^{\mathrm{i}}(x, y) \pm \phi^{\mathrm{i}}(x,-y)\right] .
$$

If the array is excited by incident wave $\phi_{ \pm}^{\mathrm{i}}(x, y)$, then the resulting coefficients $A_{m}^{p}$ and $I_{m}^{p}$ satisfy the identity

$$
\mathcal{U}_{-m}^{p}= \pm(-1)^{m} \mathcal{U}_{m}^{p}
$$

This often leads to useful simplifications, and also to an increase in performance when inverting linear systems. For brevity, we will give equations for the complete 

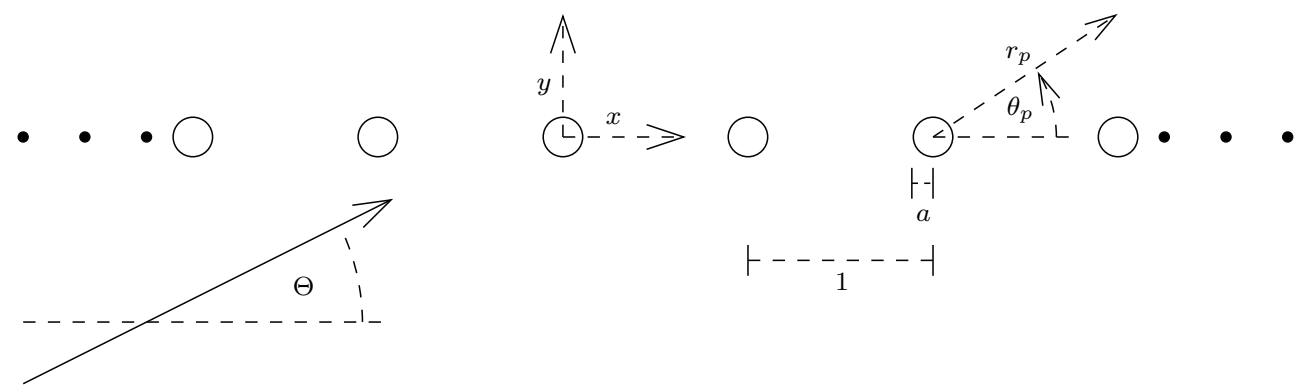

FIG. 3.1. Schematic diagram of a periodic array with circular scatterers centered at the points $(p, 0)$ in the $(x, y)$ plane, and a plane wave incident at angle $\Theta$.

wavefield, and it is to be understood that these can always be decomposed in the manner described above.

To conclude this section, we will now review the theory of periodic arrays, starting with the method for obtaining the coefficients $A_{m}^{p}(\emptyset)$ in the case where the incident field is the plane wave

$$
\phi^{\mathrm{i}}(x, y)=\mathrm{e}^{\mathrm{i} k(x \cos \Theta+y \sin \Theta)}
$$

see Figure 3.1. Values for $A_{m}^{p}(\emptyset)$ are of course required as a starting point, and the technique used to obtain them provides some motivation for the MASM developed in section 4 to solve the canonical problems. First of all, we require a second set of equations relating the coefficients $A_{m}^{p}(\emptyset)$ and $I_{m}^{p}(\emptyset)$. This will form a closed system, when combined with (3.4), and can be obtained using Graf's addition theorem [14, section 2.5]. For the specific case under consideration here, we have

$$
I_{m}^{p}(\emptyset)=\mathrm{i}^{m} \mathrm{e}^{\mathrm{i} p k \cos \Theta} \mathrm{e}^{-\mathrm{i} m \Theta}+\sum_{v} \sum_{j \neq p} A_{v}^{j}(\emptyset) X_{v-m}^{p-j} H_{v-m}^{(1)}(k|p-j|),
$$

where $X_{v}^{p}=\operatorname{sgn}(p)^{v}$. Given that the only difference between the field at the point $(x, y)$ and that at $(x+j, y), j \in \mathbb{Z}$, is a phase shift due to the incident plane wave, this can be simplified by seeking a solution for which

$$
A_{m}^{p}(\emptyset)=A_{m}^{0}(\emptyset) \mathrm{e}^{\mathrm{i} p k \cos \Theta} .
$$

Enforcing the boundary conditions via (3.4), and then making use of (3.11), we obtain

$$
A_{m}^{0}(\emptyset)+Z_{m} \sum_{v} A_{v}^{0}(\emptyset) \sigma_{v-m}(k \cos \Theta)=-Z_{m} \mathrm{i}^{m} \mathrm{e}^{-\mathrm{i} m \Theta},
$$

which is a linear system involving only an order sum. The function $\sigma_{n}(t)$ is a Schlömilch series of order $n$, i.e.,

$$
\sigma_{n}(t)=\sum_{j=1}^{\infty}\left[\mathrm{e}^{-\mathrm{i} j t}+(-1)^{n} \mathrm{e}^{\mathrm{i} j t}\right] H_{n}^{(1)}(k j) .
$$

If the values of $k$ and $\Theta$ are such that the Schlömilch series are divergent, the values for $A_{m}^{p}(\emptyset)$ can be obtained as in [12]. Note that $\sigma_{-n}(t)=(-1)^{n} \sigma_{n}(t)$.

The Schlömilch series is a type of lattice sum, and the capacity to evaluate these accurately and efficiently is crucial to the analysis of wave interactions with arrays. 
For the case under consideration here, the well-known Twersky formulae $[25,8]$ can be used. The singularity structure of $\sigma_{n}(t)$ must be considered when applying the MASM, and so we note that

$$
\sigma_{n}(t)=b_{n}(t)+2(-\mathrm{i})^{n+1}\left[\mu_{n}^{0}(t)+\sum_{j=1}^{\infty}\left(\mu_{n}^{j}(t)+\mu_{-n}^{-j}(t)-\frac{\delta_{n 0}}{\pi j}\right)\right],
$$

where $b_{n}(t)$ is an entire function that can be expressed as a finite sum of Bernoulli polynomials, and

$$
\mu_{n}^{j}(t)=\frac{[t+2 j \pi-\gamma(t+2 j \pi)]^{n}}{k^{n} \gamma(t+2 j \pi)}
$$

The function $\gamma(t)$ is defined for real $t$ via

$$
\gamma(t)=\left\{\begin{aligned}
\sqrt{t^{2}-k^{2}} & :|t| \geq k \\
-\mathrm{i} \sqrt{k^{2}-t^{2}} & :|t|<k
\end{aligned}\right.
$$

For $n=0,1$, and 2, the summand in (3.14) is $O\left(j^{-3}\right)$ as $j \rightarrow \infty$; for larger $n$ it is $O\left(j^{-5}\right)$ or smaller. The rate of convergence can easily be accelerated by expanding the summand in (3.14) for large $j$. Where derivatives are required, the formula

$$
\frac{\mathrm{d} \mu_{n}^{j}}{\mathrm{~d} t}=\frac{-\mu_{n}^{j}(t)}{\gamma(t+2 j \pi)}\left[n+\frac{t+2 j \pi}{\gamma(t+2 j \pi)}\right]
$$

can be used. The infinite summation in the resulting formula for $\sigma_{n}^{\prime}(t)$ has a summand that is $O\left(j^{-3}\right)$ as $j \rightarrow \infty$ for $n=0$ and $n=1$, and $O\left(j^{-5}\right)$ or smaller for larger values of $n$. Again, the convergence can be accelerated where necessary.

An important property of infinite periodic arrays is their capacity to support RB surface waves in some circumstances. These propagate without loss along the array and decay exponentially in other directions. The presence of RB waves corresponds to the existence of nontrivial homogeneous solutions to the periodic array problem with the form

$$
\phi_{R B}^{\mathrm{t}}(\mathbf{r})=\sum_{m} \sum_{p} \widetilde{B}_{m} \mathrm{e}^{\mathrm{i} p \widetilde{\beta}} H_{m}^{(1)}\left(k r_{p}\right) \mathrm{e}^{\mathrm{i} m \theta_{p}}
$$

where $\widetilde{\beta} \in \mathbb{R}$ is an arbitrary phase shift. The coefficients $\widetilde{B}_{m}$ satisfy the same system of equations as $A_{m}^{0}(\emptyset)$ (i.e., (3.12)), but with the right-hand side set to zero and $k \cos \Theta$ replaced by $\widetilde{\beta}$; thus

$$
\widetilde{B}_{m}+Z_{m} \sum_{v} \widetilde{B}_{v} \sigma_{v-m}(\widetilde{\beta})=0
$$

in which $\widetilde{B}_{m} \neq 0$ for at least one $m$. A straightforward method for finding the appropriate values for $\widetilde{\beta}$ is given in [6]. The associated coefficients $\widetilde{B}_{m}$ are then normalized so that

$$
\sum_{m}\left|\widetilde{B}_{m}\right|^{2}=1
$$

Copyright $@$ by SIAM. Unauthorized reproduction of this article is prohibited. 
Given the evident $2 \pi$-periodicity of the Schlömilch series (3.13), distinct solutions to (3.19) can occur only for $\widetilde{\beta} \in[0,2 \pi)$. Full details of the parameter ranges for which RB modes have been found are given in [23]. Here we summarize the important details.

If the surface of the scatterers is subject to a Dirichlet boundary condition, then RB waves do not occur [2]. On the other hand, if a Neumann boundary condition is in use, then up to two distinct modes are known to exist. One of these is symmetric about $y=0$; this can occur for scatterers of any size, for a range of wavenumbers $0<k<k_{\max }^{\mathrm{s}}<\pi$. The other is an antisymmetric mode which exists in the range $k_{\min }^{\mathrm{a}}<k<k_{\max }^{\mathrm{a}}<\pi$, but only if $a \gtrsim 0.403$. The cut-off values depend upon the scatterer radius $a$. Outside the given ranges for $k$, the $\mathrm{RB}$ wave is replaced by a mode that is evanescent in $x$. In both the symmetric and antisymmetric cases, the principal value for $\widetilde{\beta}$ lies in the interval $(k, \pi)$ and corresponds to a right-propagating wave. The associated left-propagating mode has the phase shift $2 \pi-\widetilde{\beta}$ in place of $\widetilde{\beta}$ and the coefficient $(-1)^{m} \widetilde{B}_{m}$ in place of $\widetilde{B}_{m}$. As $k \rightarrow k_{\max }, \widetilde{\beta} \rightarrow \pi$, i.e., the RB modes become standing waves. The amplitude with which RB modes are excited is a key parameter in the solution, and obtaining this is a major goal of our analysis. In what follows, we will assume that exactly one type of RB mode occurs (i.e., symmetric or antisymmetric). It is not difficult to modify our subsequent analysis if this is not the case. In a problem where the incident wave has been decomposed using (3.7), there is at most one mode for each component of the solution.

4. Canonical problems. In order to proceed, we must determine $\psi_{n}^{0}$, i.e., the total field that occurs when scatterer 0 is replaced by a unit source of order $n$. In this case, we have the expansion

$$
\psi_{n}^{0}(\mathbf{r})=\sum_{m} \sum_{p} C_{m, n}^{p, 0} H_{m}^{(1)}\left(k r_{p}\right) \mathrm{e}^{\mathrm{i} m \theta_{p}},
$$

where

$$
C_{m, n}^{0,0}=\delta_{m n},
$$

as in (2.12). A useful symmetry relation can be obtained by changing $x$ to $-x$ and $y$ to $-y$ (and therefore $r_{p} \rightarrow r_{-p}$ and $\theta_{p} \rightarrow \pi+\theta_{-p}$ ) in (4.1). After applying (4.2) and comparing the result to (4.1), we find that

$$
C_{m, n}^{-p, 0}=(-1)^{m+n} C_{m, n}^{p, 0} .
$$

As before, a linear system for the unknown coefficients can be obtained by locally expanding $\psi_{n}^{0}$ about the point $r_{p}=0$; thus

$$
\psi_{n}^{0}\left(r_{p}, \theta_{p}\right)=\sum_{m}\left[K_{m, n}^{p, 0} J_{m}\left(k r_{p}\right)+C_{m, n}^{p, 0} H_{m}^{(1)}\left(k r_{p}\right)\right] \mathrm{e}^{\mathrm{i} m \theta_{p}} .
$$

An expression for the incoming field coefficients $K_{m, n}^{p, 0}$ in terms of the outgoing coefficients $C_{m, n}^{p, 0}$ can be deduced from (3.10) by simply omitting the term due to plane wave forcing. We find that

$$
K_{m, n}^{p, 0}=\sum_{v} \sum_{j \neq p} C_{v, n}^{j, 0} X_{v-m}^{p-j} H_{v-m}^{(1)}(k|p-j|),
$$

and the boundary condition gives

$$
C_{m, n}^{p, 0}+Z_{m} K_{m, n}^{p, 0}=0, \quad p \neq 0 .
$$


The MASM can now be used to obtain an expression for $C_{m, n}^{p, 0}$. The principal idea is derived from the original array scanning method [27, 16, 23], in which the unknown coefficients are represented as Fourier integrals. First of all, introduce damping by writing

$$
k=\operatorname{Re}[k]+\mathrm{i} \epsilon,
$$

where $\epsilon>0$. This ensures the convergence of the summations over the spatial index in subsequent equations. Once the solutions are obtained, we can take the limit $\epsilon \rightarrow 0$ to retrieve the time-harmonic field. Next, define the function $f_{m, n}(t)$ by writing

$$
f_{m, n}(t)=\mathrm{i} \sum_{p} C_{m, n}^{p, 0} \mathrm{e}^{-\mathrm{i} p t}
$$

so that we have

$$
C_{m, n}^{p, 0}=\frac{1}{2 \pi \mathrm{i}} \int_{0}^{2 \pi} f_{m, n}(t) \mathrm{e}^{\mathrm{i} p t} \mathrm{~d} t .
$$

One motivation for this choice of representation is that the spatial dependence of the integral is such that if we substitute (4.9) into (4.5), the sum over $j$ will become a Schlömilch series as in (3.13). Indeed, combining (4.5), (4.6), and (4.9), we find that

$$
\int_{0}^{2 \pi}\left[f_{m, n}(t)+Z_{m} \sum_{v} f_{v, n}(t) \sigma_{v-m}(t)\right] \mathrm{e}^{\mathrm{i} p t} \mathrm{~d} t=0, \quad p \neq 0 .
$$

A second motivation for (4.8) is that the integration in (4.9) facilitates a simple means by which the left-hand side of (4.10) can be made to vanish for all $p \neq 0$. If we now write

$$
f_{m, n}(t)+Z_{m} \sum_{v} f_{v, n}(t) \sigma_{v-m}(t)=\mathcal{F}_{m, n}(t),
$$

then (4.10) becomes

$$
\int_{0}^{2 \pi} \mathcal{F}_{m, n}(t) \mathrm{e}^{\mathrm{i} p t} \mathrm{~d} t=0, \quad p \neq 0 .
$$

By considering the Fourier series expansions of $\mathcal{F}_{m, n}(t)$, it becomes clear that (4.11) can be satisfied if and only if these functions are constants, which we denote by $\mathcal{F}_{m, n}$. The values for these are fixed by setting $p=0$ in (4.9) and imposing the requirement (4.2); hence

$$
\frac{1}{2 \pi \mathrm{i}} \int_{0}^{2 \pi} f_{m, n}(t) \mathrm{d} t=\delta_{m n} .
$$

Note that the system of equations (4.11) contains only an order sum, and also that the source order $n$ does not affect the operator on the left-hand side, which is of exactly the same form as those appearing in (3.12) and (3.19), with the variable $t$ taking the place of the parameters $k \cos \Theta$ and $\widetilde{\beta}$.

In order to determine the coefficients $\mathcal{F}_{m, n}$, we introduce the function $g_{m, n}(t)$ as the solution to the linear system (4.11), but with the right-hand side replaced by $\delta_{m n}$, i.e.,

$$
g_{m, n}(t)+Z_{m} \sum_{v} g_{v, n}(t) \sigma_{v-m}(t)=\delta_{m n} .
$$

Copyright (C) by SIAM. Unauthorized reproduction of this article is prohibited. 
Since the right-hand side is known, this system of equations can be inverted numerically for any value of $t$ at which both $\sigma_{n}(t)$ and $g_{v, n}(t)$ are analytic. If (4.14) is multiplied by $\mathcal{F}_{n, u}$ and then summed over all integers $n$, we see that $g_{m, n}(t)$ is related to $f_{m, n}(t)$ via

$$
\sum_{v} g_{m, v}(t) \mathcal{F}_{v, n}=f_{m, n}(t)
$$

Integrating (4.15) yields

$$
\frac{1}{2 \pi \mathrm{i}} \sum_{v} \mathcal{F}_{v, n} \int_{0}^{2 \pi} g_{m, v}(t) \mathrm{d} t=\delta_{m n},
$$

in view of (4.13). In principle, therefore, the solutions to the canonical problems are now available - take the limit $\epsilon \rightarrow 0$ in (4.7) and then apply quadrature to compute the integrals in (4.16). This latter step is discussed in the appendix. This done, the resulting linear system can be inverted to yield $\mathcal{F}_{m, n}$. However, taking the limit $\epsilon \rightarrow 0$ in (4.7) will cause singularities to appear on the real line, and so we must determine the correct indentations for the path of integration.

First, for any $k>0$, there exists $\lambda \in \mathbb{Z}$ such that $\operatorname{Re}\left[k_{\lambda}\right] \in[0,2 \pi]$, where

$$
k_{\lambda}=k+2 \lambda \pi .
$$

Equation (3.14) shows that the function $\sigma_{n}(t)$ has a branch point at $t=k_{\lambda}$; another is located at $t=2 \pi-k_{\lambda}$. Note that $\operatorname{Im}\left[k_{\lambda}\right]=\epsilon$, and $\operatorname{Im}\left[2 \pi-k_{\lambda}\right]=-\epsilon$. The functions $f_{m, n}(t)$ and $g_{m, n}(t)$ will inherit these singularities via (4.11) and (4.14), respectively. The special case in which $k_{\lambda}=2 \pi-k_{\lambda}=\pi$ can be handled by adjusting the path of integration in (4.9) to run from $-\pi$ to $\pi$.

A second important possibility is that, after taking the limit $\epsilon \rightarrow 0$ in (4.7), there may exist real values of $t$ at which the matrix of known coefficients appearing on the left-hand side of (4.11) and (4.14) is singular. These correspond to the existence of $\mathrm{RB}$ waves, as discussed in section 3 ; at $t=\widetilde{\beta}$ the left-hand side of (4.11) (and also (4.14)) is identical to that of (3.19). In general, the Fredholm alternative permits solutions at $t=\widetilde{\beta}$ and $t=2 \pi-\widetilde{\beta}$ if the functions $f_{m, n}(t)$ and $g_{m, n}(t)$ possess simple poles at these points. Numerical results in [6] show that $\mathrm{d} \widetilde{\beta} / \mathrm{d} k>0$, and so when we add damping using (4.7) the pole at $t=\widetilde{\beta}$ moves above the real line, and that at $t=2 \pi-\widetilde{\beta}$ moves below. If we now let $\epsilon \rightarrow 0$ in (4.7) so as to retrieve the timeharmonic solution, we find that the correct indentations for the path of integration are those shown in Figure 4.1. This is the only configuration that leads to a purely outgoing scattered field in the limit $\sqrt{x^{2}+y^{2}} \rightarrow \infty$. The residues at the poles of $f_{m, n}(t)$ determine the amplitudes of any RB waves that are excited, and these make a contribution to $C_{m, n}^{p, 0}$ that does not decay in the limit $|p| \rightarrow \infty$. We now calculate these, using the method in [23]. First, multiply (4.11) by $t-\widetilde{\beta}$ and then take the limit $t \rightarrow \widetilde{\beta}$. The residue of the function $f_{m, n}(t)$ at the pole must satisfy the resulting homogeneous linear system, which is identical to (3.19), and hence

$$
\operatorname{Res}_{t=\widetilde{\beta}} f_{m, n}(t)=c_{n} \widetilde{B}_{m}
$$

for some constant $c_{n}$. Essentially, the coefficients $\widetilde{B}_{m}$ describe the shape of the RB wave, and $c_{n}$ is the amplitude. The same procedure can then be applied with $\widetilde{\beta}$ 


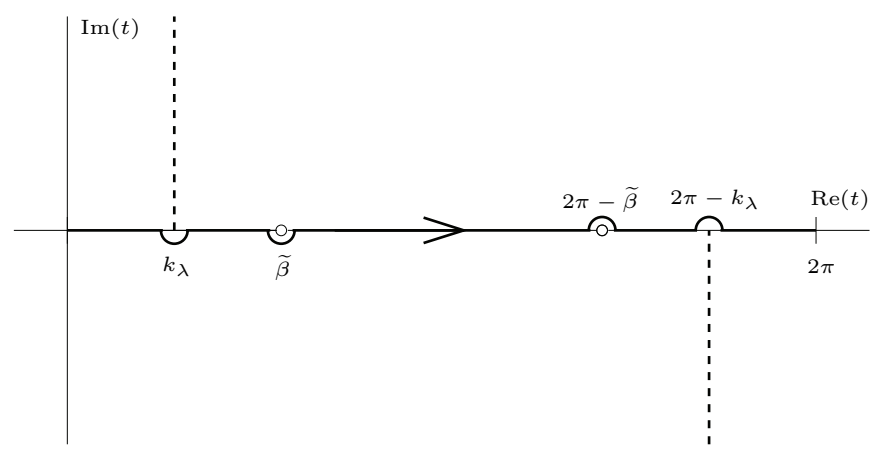

FIG. 4.1. Singularity structure and indented contour of integration in the $t$ plane. The poles at $t=\widetilde{\beta}$ and $t=2 \pi-\widetilde{\beta}$ do not occur if Dirichlet boundary conditions are imposed on the surface of the regular scatterers, or if $\lambda \neq 0$ (i.e., $k>\pi$ ).

replaced by $2 \pi-\widetilde{\beta}$, (and $\widetilde{B}_{m}$ by $(-1)^{m} \widetilde{B}_{m}$ ) and the symmetry relation (4.3) then shows that

$$
f_{m, n}(t)=\widehat{f}_{m, n}(t)+c_{n} \widetilde{B}_{m}\left[\frac{1}{t-\widetilde{\beta}}-\frac{(-1)^{m+n}}{t+\widetilde{\beta}-2 \pi}\right]
$$

where $\widehat{f}_{m, n}(t)$ is analytic at $t=\widetilde{\beta}$ and $t=2 \pi-\widetilde{\beta}$. Finally, substitute (4.19) into (4.11), transfer the terms with denominator $t-\widetilde{\beta}$ to the right-hand side, and take the limit $t \rightarrow \widetilde{\beta}$ using L'Hôpital's rule as appropriate. We can now apply the Fredholm alternative to the resulting linear system. The left-hand side consists of a singular matrix, multiplied by a vector of bounded functions. Therefore, a solution can exist if and only if the right-hand side is orthogonal to the (nontrivial) solution to the homogeneous adjoint problem [20, eqns. (5.7)-(5.9)]. The latter is easily shown to be $\widetilde{B}_{m} / Z_{m}^{*}[23]$, leading to the following equation for $c_{n}$ :

$$
\sum_{m} \frac{\widetilde{B}_{m}^{*} \mathcal{F}_{m, n}}{Z_{m}}=c_{n} \sum_{m} \widetilde{B}_{m}^{*} \sum_{v} \widetilde{B}_{v} \sigma_{v-m}^{\prime}(\widetilde{\beta}) .
$$

Here, the superscript " $*$ " denotes the complex conjugate, and the prime a derivative with respect to the argument. The residues of the function $g_{m, n}(t)$ can be calculated in exactly the same way; simply replace $f$ with $g$ in (4.18) and (4.19) and $\mathcal{F}_{m, n}$ with $\delta_{m n}$ in (4.20).

The asymptotic behavior of $C_{m, n}^{p, 0}$ in the limit $|p| \rightarrow \infty$ can be obtained by noting that $f_{m, n}(t)$ is $2 \pi$-periodic, this property being inherited from the Schlömilch series via (4.11). Consequently, if the path of integration in (4.9) is closed in the upper half plane, the contributions from $t=\mathrm{i} u$ and $t=2 \pi+\mathrm{i} u, u>0$, cancel each other. Therefore, as $p \rightarrow \infty$, we have

$$
C_{m, n}^{p, 0} \sim c_{n} \widetilde{B}_{m} \mathrm{e}^{\mathrm{i} p \widetilde{\beta}}+C_{m, n} \frac{\mathrm{e}^{\mathrm{i} k p}}{p^{3 / 2}}+O\left(p^{-5 / 2}\right) .
$$

Here, the second term on the right-hand side is the dominant contribution from the branch point at $t=k_{\lambda}$. The dependence upon $p$ can be deduced by using the method in [12] to show that $f_{m, n}(t)$ remains finite as $t \rightarrow k_{\lambda}$. Given that $t=k_{\lambda}$ is a branch point of square root type, the result follows. In principle, one can also obtain a formula 
for the coefficient $C_{m, n}$ using a similar technique, but this is somewhat involved. The behavior of $C_{m, n}^{p, 0}$ in the limit $p \rightarrow-\infty$ can be deduced by closing the contour of integration in (4.9) in the lower half plane; alternatively, the symmetry relation (4.3) can be used.

A final point concerns the field incoming toward the source, which must be calculated if we are dealing with defects that do not consist of absent scatterers. From (4.4) it is seen that this amounts to finding the value of $K_{m, n}^{0,0}$, which can be achieved by setting $p=0$ in (4.5). Both the spatial sum and the order sum can be evaluated exactly. Thus, on using (4.9), we have

$$
K_{m, n}^{0,0}=\frac{1}{2 \pi \mathrm{i}} \int_{0}^{2 \pi} \sum_{v} f_{v, n}(t) \sigma_{v-m}(t) \mathrm{d} t .
$$

Equation (4.11) reduces this to an integral whose value is known in view of (4.13), the result being

$$
Z_{m} K_{m, n}^{0,0}=-\delta_{m n}-\mathrm{i} \mathcal{F}_{m, n} .
$$

5. Illustrative results. In this section we present some numerical results for a variety of different situations. We also show how the interaction theory for defects can be combined with the infinite array subtraction technique developed in [11], and the large array approximation used in [24] to validate results, and extend the range of applicability. Particular attention is paid to the determination of the amplitude with which RB waves are excited by the defects. Accurate computation by more direct numerical methods is difficult (see the appendix, and also [11]), but our approach is numerically efficient, and we are able to compute the amplitudes for all possible $k$ and $\Theta$. In view of the number of cases that can be solved, we have not carried out a comprehensive parameter survey, but instead we have attempted to provide a representative sample of the types of result that can be obtained. In performing any such calculations, the rapidly convergent order summations that occur throughout our analysis must be truncated at some suitable value, which depends on the size of $k a$. This must be chosen to be large enough to yield accurate results, but not so large as to unnecessarily increase program execution time or generate near singular linear systems. The truncation levels used by our numerical codes are the same as those reported in [24]. Unless otherwise stated, Neumann boundary conditions are applied on the surface of the regular scatterers.

5.1. Localized defects. In cases where the defects are confined to a small section of the array, all of the relevant integrals can easily be evaluated by quadrature. The asymptotic behavior of $A_{m}^{p}(\mathcal{D})$ for large $p$ can be obtained using $(2.16),(2.17)$, (3.11), and (4.21). A formula for large, negative $p$ can be obtained in a similar way by applying the symmetry relation (4.3) in (2.17). We find that, as $p \rightarrow \pm \infty$,

$$
A_{m}^{p}(\mathcal{D}) \sim A_{m}^{0}(\emptyset) \mathrm{e}^{\mathrm{i} k p \cos \Theta}+( \pm 1)^{m} \Gamma^{ \pm} \widetilde{B}_{m} \mathrm{e}^{\mathrm{i}|p| \widetilde{\beta}}+O\left(|p|^{-3 / 2}\right)
$$

where

$$
\Gamma^{ \pm}=\sum_{n}( \pm 1)^{n} c_{n} \sum_{q \in \mathcal{D}} a_{n}^{q} \mathrm{e}^{\mp \mathrm{i} q \widetilde{\beta}}
$$

The quantity $\Gamma^{+}\left(\Gamma^{-}\right)$is the complex amplitude coefficient of the right- (left-) propagating $\mathrm{RB}$ wave that is excited by the defect. This depends upon the solutions to 
(a)

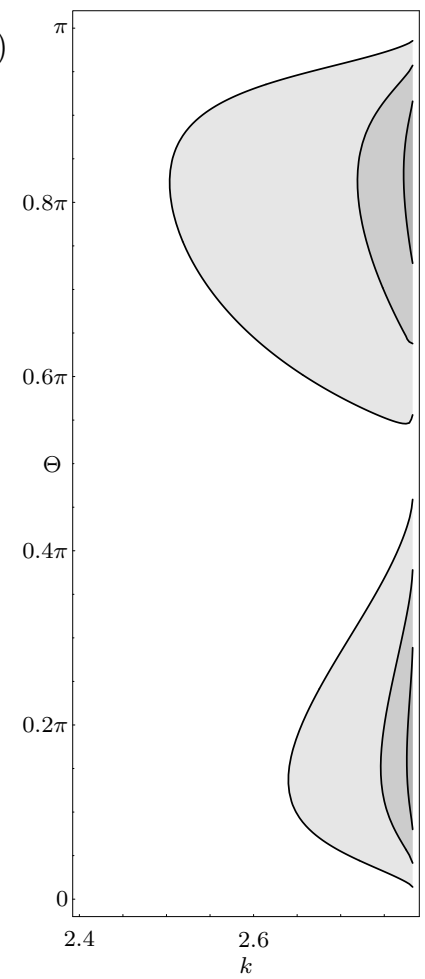

(b)

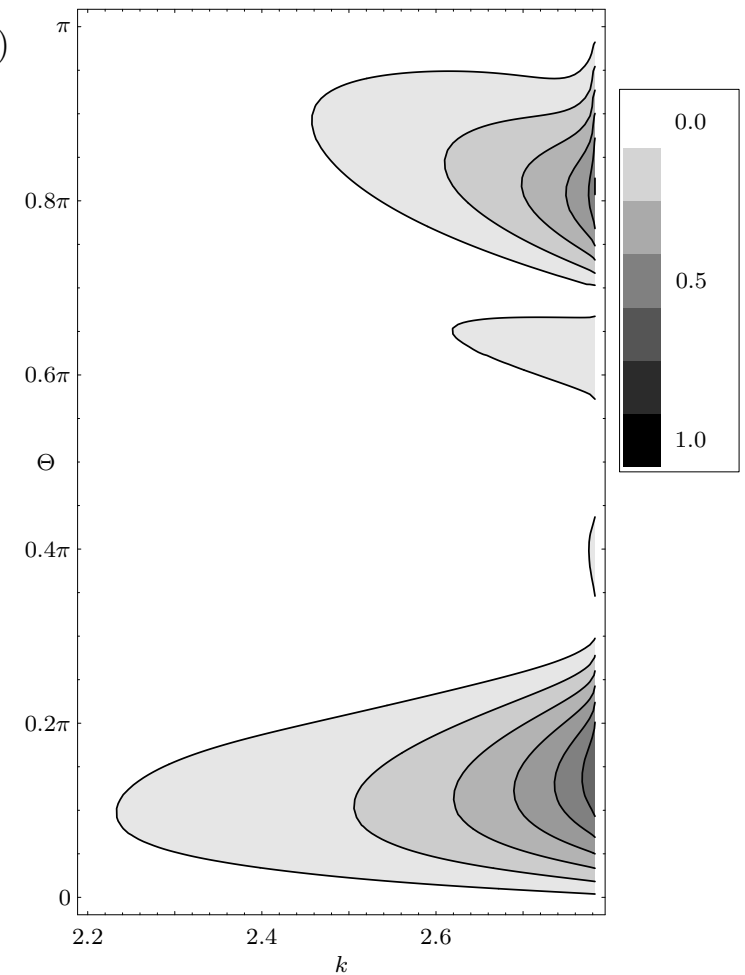

FIG. 5.1. Contour plots showing the amplitude of the right-propagating $R B$ wave, $\left|\Gamma^{+}\right|$, for $a=0.25$ with varying $k$ and $\Theta$. (a) $\mathcal{D}=\{0\}$. (b) $\mathcal{D}=\{0,1,2,3,4,5\}$.

the canonical problems and the interactions between the defects via the coefficients $c_{n}$, and the sum over $\mathcal{D}$, respectively.

Figure 5.1 shows contour plots of $\left|\Gamma^{+}\right|$with varying $k$ and $\Theta$, for $a=0.25$ and two different defect sets: $\mathcal{D}=\{0\}$ and $\mathcal{D}=\{0,1,2,3,4,5\}$. Figure 5.2 shows similar plots, but for the antisymmetric RB wave on an array with $a=0.49$. In all cases, $\left|\Gamma^{-}\right|$can be deduced by symmetry. The computation time required to obtain data for figures such as these is greatly reduced by the fact that the canonical problems need only be solved once for each value of $k$. The general trend for the amplitude to increase with $k$ is consistent with the cases of excitation at an array end [11], and by an aperiodic source [23]. The upper limit for $k$ is the cut-off $(k \approx 2.783$ for $a=0.25$ and $k \approx 2.971$ for the antisymmetric mode on an array with $a=0.49$ ), above which the RB waves cease to exist. For all values of $k$ smaller than those shown, the symmetric mode exists but is excited at a very low amplitude. The antisymmetric mode does not exist for $k \lesssim 1.796$; for intermediate values up to those that are shown in Figure 5.2 the excitation amplitude is small. The dependence of $\Gamma^{+}$upon the angle of incidence $\Theta$ exhibits a number of interesting features. First, the surface wave is cut off completely as $\Theta \rightarrow 0$ and $\Theta \rightarrow \pi$. In fact, the total field vanishes in these limits, as demonstrated in [12]; the presence of a finite set of defects has no bearing on this. The cut-off at $\Theta=0$ is sharper in Figures 5.1(b) and 5.2(b) than it is in Figures 5.1(a) and 5.2(a); this is consistent with the case of excitation at the end of a semiinfinite array, where the cut-off disappears, and the amplitude is generally greatest at head-on incidence [11]. The two-peak structure, and the fact that the relative size 
(a)

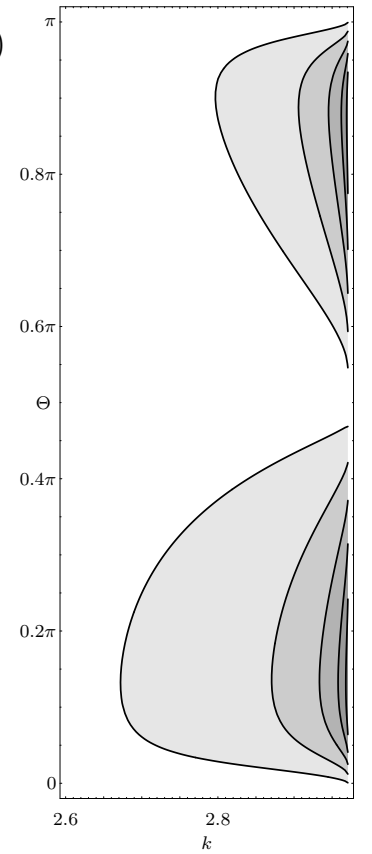

(b)

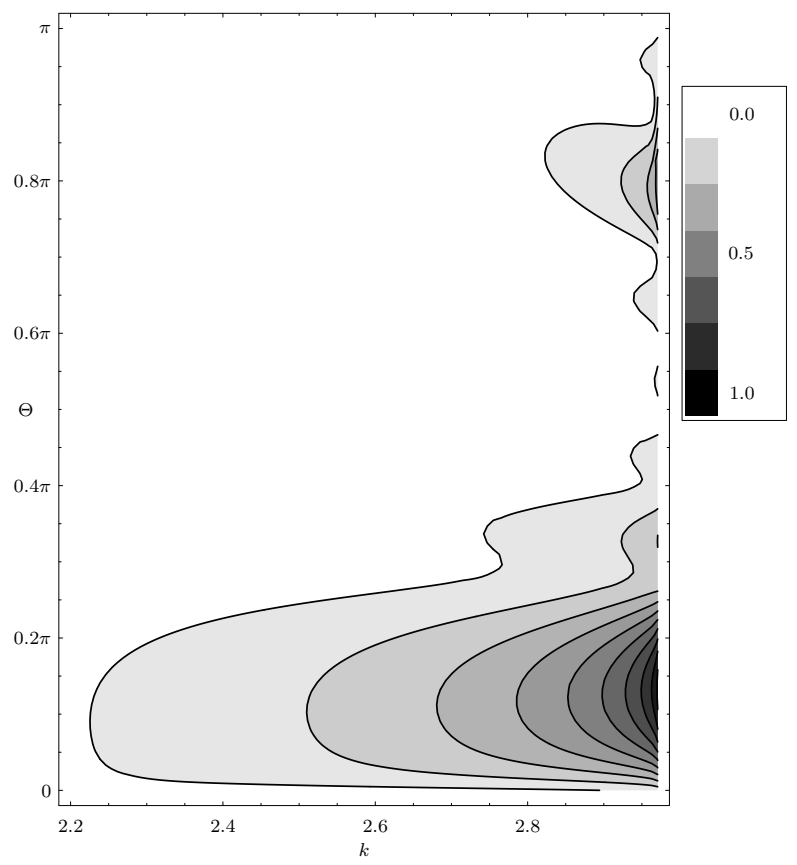

FIG. 5.2. Contour plots showing the amplitude of the right propagating antisymmetric $R B$ wave, $\left|\Gamma^{+}\right|$, for $a=0.49$ with varying $k$ and $\Theta$. (a) $\mathcal{D}=\{0\}$, (b) $\mathcal{D}=\{0,1,2,3,4,5\}$.

of the peak at $\Theta \approx 0.8 \pi$ is reduced for the larger defect sets, is also consistent with the case of excitation at an end. Finally, note that for the case where $\mathcal{D}=\{0\}$, the amplitude of the symmetric right-propagating $\mathrm{RB}$ wave is largest when $\Theta \approx 0.8 \pi$, which corresponds to an incident field whose $x$-component is propagating to the left.

The infinite array subtraction methods introduced in [11] provide a useful means of validating results such as those shown in Figures 5.1 and 5.2. If we write

$$
D_{m}^{p}(\mathcal{D})=A_{m}^{p}(\mathcal{D})-A_{m}^{p}(\emptyset)
$$

then, on recalling that $A_{m}^{p}(\mathcal{D})=0$ for $p \in \mathcal{D}$, it is not difficult to use the results in section 3 to show that the coefficients $D_{m}^{p}(\mathcal{D})$ satisfy the linear system of equations

$$
\begin{aligned}
D_{m}^{p}(\mathcal{D})+Z_{m} \sum_{v} & \sum_{\substack{j \notin \mathcal{D} \\
j \neq p}} D_{v}^{j}(\mathcal{D}) X_{v-m}^{p-j} H_{v-m}^{(1)}(k|p-j|) \\
& =Z_{m} \sum_{v} A_{v}^{0}(\emptyset) \sum_{j \in \mathcal{D}} \mathrm{e}^{\mathrm{i} j k \cos \Theta} X_{v-m}^{p-j} H_{v-m}^{(1)}(k|p-j|), \quad p \notin \mathcal{D} .
\end{aligned}
$$

Note that the right-hand side has been simplified using (3.4) (with $\mathcal{D}=\emptyset$ ) and (3.10). If no $\mathrm{RB}$ waves are present, then we should expect that $D_{p} \rightarrow 0$ as $|p| \rightarrow \infty$. On the other hand, if RB waves are present in the solution, their contribution can be isolated by writing

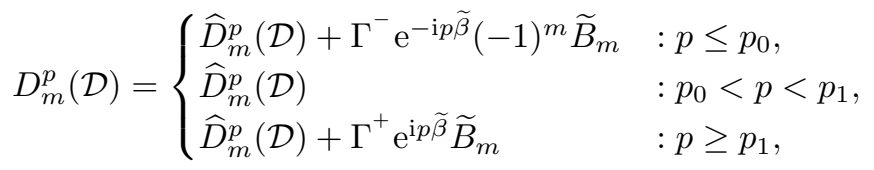


where $p_{0}$ and $p_{1}$ are chosen so that the array is regular for $p \leq p_{0}$ and $p \geq p_{1}$. Substituting this into (5.4), we find that the coefficients $\widehat{D}_{m}^{p}(\mathcal{D})$ satisfy a linear system which has the same left-hand side as (5.4) and correction terms $L_{m}^{p}$ and $R_{m}^{p}$ (due to the left- and right-propagating RB waves, respectively) added to the right-hand side. A straightforward calculation shows that

$$
L_{m}^{p}= \begin{cases}-\Gamma^{-} Z_{m} \mathrm{e}^{-\mathrm{i} p \widetilde{\beta}} \sum_{v}(-1)^{v} \widetilde{B}_{v} S_{v-m}^{p-p_{0}}(\widetilde{\beta}) & : p>p_{0}, \\ \Gamma^{-}(-1)^{m} Z_{m} \mathrm{e}^{-\mathrm{i} p \widetilde{\beta}} \sum_{v} \widetilde{B}_{v} S_{v-m}^{1+p_{0}-p}(-\widetilde{\beta}) & : p \leq p_{0}\end{cases}
$$

and

$$
R_{m}^{p}= \begin{cases}-\Gamma^{+} Z_{m} \mathrm{e}^{\mathrm{i} p \widetilde{\beta}} \sum_{v} \widetilde{B}_{v} S_{m-v}^{p_{1}-p}(\widetilde{\beta}) & : p<p_{1}, \\ \Gamma^{+} Z_{m} \mathrm{e}^{\mathrm{i} p \widetilde{\beta}} \sum_{v} \widetilde{B}_{v} S_{v-m}^{1+p-p_{1}}(-\widetilde{\beta}) & : p \geq p_{1},\end{cases}
$$

where

$$
S_{m}^{p}(\beta)=\sum_{j \geq p} \mathrm{e}^{\mathrm{i} j \beta} H_{m}^{(1)}(k j)
$$

this half range Schlömilch series can be efficiently computed using methods in [8]. The fact that the RB wave is a homogeneous solution to the periodic array problem has been used to simplify $L_{m}^{p}$ for $p \leq p_{0}$ and $R_{m}^{p}$ for $p \geq p_{1}$. If we now solve the linear system for $\widehat{D}_{m}^{p}(\mathcal{D})$, the solution will decay as $|p| \rightarrow \infty$, but only if the correct values for the RB amplitudes $\Gamma^{-}$and $\Gamma^{+}$are used.

As an example, consider the parameter set $a=0.25, k=2.5, \Theta=0.1 \pi$, and $\mathcal{D}=\{0\}$, which is included in Figure 5.1. Figure 5.3 shows a logarithmic plot of $D_{p}$ for this case, where

$$
D_{p}=\sum_{m}\left|D_{m}^{p}(\mathcal{D})\right|^{2}
$$

This provides a simple measure of the difference between the scattered fields in the periodic and defective array problems. The data are obtained by truncating the system (5.4) at $|p|=100$. Clearly, $D_{p}$ does not decay as $|p| \rightarrow \infty$; instead it oscillates about a fixed value corresponding to the amplitude of the RB wave. As in Figure 5.1 , this is stronger to the left of the defect. The quantity $\widehat{D}_{p}$ is also plotted in Figure 5.3. This is obtained by replacing $D_{m}^{p}(\mathcal{D})$ with $\widehat{D}_{m}^{p}(\mathcal{D})$ in $(5.9)$. Values for $\Gamma^{-}(\approx 0.07613+0.02638 \mathrm{i})$ and $\Gamma^{+}(\approx-0.04897+0.00176 \mathrm{i})$ are obtained using $(2.15)$ and (5.2), with the canonical problems solved using the MASM. These values are then used in (5.6) and (5.7). The fact that $\widehat{D}_{p}$ decays as $|p|$ is increased confirms that these amplitudes are indeed correct.

5.2. Irregular scatterers. In cases where the defects do not consist of absent scatterers, we must close the system of equations for $a_{m}^{p}$ (2.18) by applying boundary conditions on the surface of the irregular array elements. We will assume that the irregular scatterers differ from the other array elements in either size, surface composition, or possibly both. In such cases, we can impose the boundary condition for the 


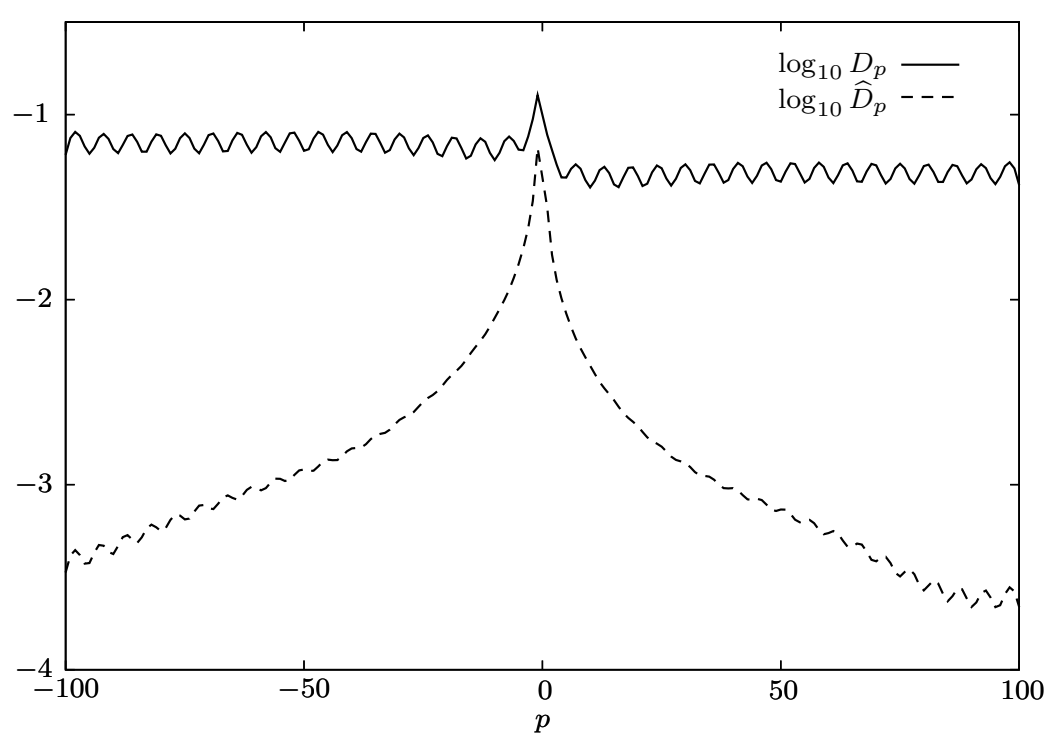

FIG. 5.3. Logarithmic plot of $D_{p}$ and $\widehat{D}_{p}$ for $a=0.25, k=2.5, \Theta=0.1 \pi$, and $\mathcal{D}=\{0\}$.

irregular scatterers using an equation similar to (3.4), but with a different scattering coefficient $\widehat{Z}_{m}$; thus

$$
A_{m}^{p}(\mathcal{D})+\widehat{Z}_{m} I_{m}^{p}(\mathcal{D})=0, \quad p \in \mathcal{D} .
$$

As before, it is not difficult to incorporate transfer matrices so as to deal with scatterers of a different shape. Equation (5.10) is to be used in conjunction with

$$
A_{m}^{p}(\mathcal{D})=A_{m}^{p}(\emptyset)+\sum_{n} \sum_{q \in \mathcal{D}} a_{n}^{q} C_{m, n}^{p-q, 0},
$$

which is obtained from (2.12) and (2.16)-(2.18), and is valid for all $p$. The simplest way to proceed is to deduce an expression for $I_{m}^{p}(\mathcal{D})$ from (3.10) by replacing $\emptyset$ with $\mathcal{D}$. If we then use (5.11) to decompose $A_{m}^{p}(\mathcal{D})$, the spatial sums in the resulting expression can be evaluated using (3.10) and (4.5), leading to

$$
I_{m}^{p}(\mathcal{D})=I_{m}^{p}(\emptyset)+\sum_{n} \sum_{q \in \mathcal{D}} a_{n}^{q} K_{m, n}^{p-q, 0}
$$

The incoming field coefficients $K_{m, n}^{p, 0}$ on the right-hand side can then be eliminated using (3.4) (with $\mathcal{D}=\emptyset$ ), (4.6), and (4.23). This amounts to exploiting the fact that (5.11) decomposes $A_{m}^{p}(\mathcal{D})$ into contributions from fields that satisfy the boundary condition for a regular scatterer at $r_{p}=a$ and contributions for which the local expansion of the incoming field is known from (4.23). We find that

$$
-Z_{m} I_{m}^{p}(\mathcal{D})=A_{m}^{p}(\emptyset)+\sum_{n} \sum_{q \in \mathcal{D}} a_{n}^{q} C_{m, n}^{p-q, 0}+\mathrm{i} \sum_{n} a_{n}^{p} \mathcal{F}_{m, n}
$$

Finally, we can form a closed system for $a_{m}^{p}$ by combining (5.13) with (5.10) and (5.11). The resulting expression is

$$
-\mathrm{i} \widehat{Z}_{m} \sum_{n} a_{n}^{p} \mathcal{F}_{m, n}+\left(Z_{m}-\widehat{Z}_{m}\right) \sum_{n} \sum_{q \in \mathcal{D}} a_{n}^{q} C_{m, n}^{p-q, 0}=\left(\widehat{Z}_{m}-Z_{m}\right) A_{m}^{p}(\emptyset), \quad p \in \mathcal{D} .
$$


(a)

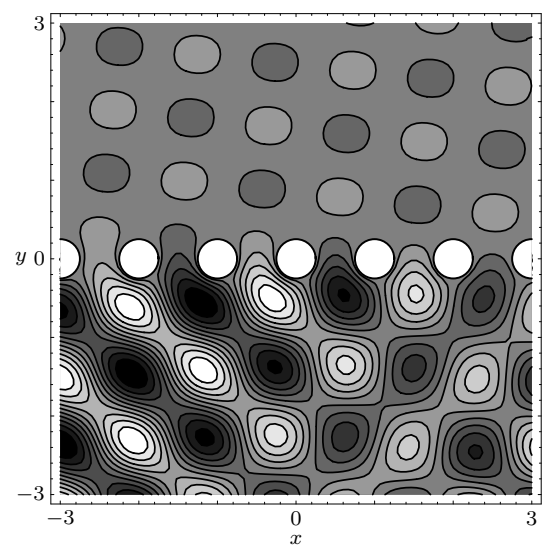

(b)

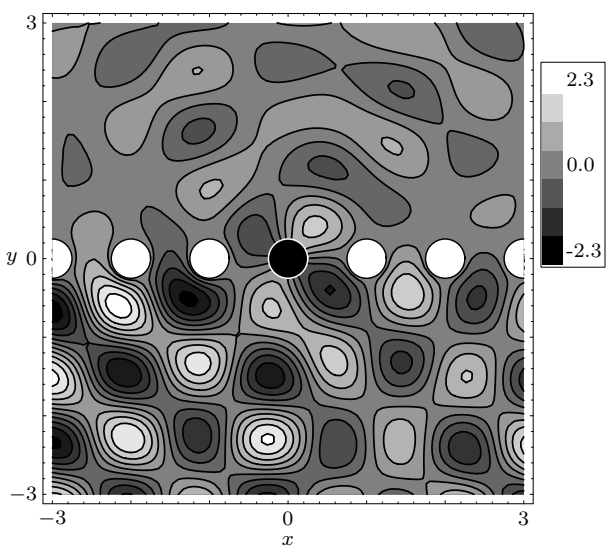

FIG. 5.4. Contour plots showing $\operatorname{Re}\left[\phi^{t}\right]$ for $a=0.25, k=5.0$, and $\Theta=0.25 \pi$, with Dirichlet boundary conditions applied on the surface of the regular scatterers. (a) $\mathcal{D}=\emptyset$. (b) $\mathcal{D}=\{0\}$. The defect is a Neumann scatterer, also with $a=0.25$.

As before, this determines the values of $a_{m}^{p} ;(5.11)$ can then be used to find values of $A_{m}^{p}(\mathcal{D})$ for $p \notin \mathcal{D}$. Note that taking $\widehat{Z}_{m}=0$ returns the equation for absent scatterers, and $\widehat{Z}_{m}=Z_{m}$ yields $a_{m}^{p}=0$, as we should expect, since then there are no defects.

Figure 5.4 shows contour plots depicting the local effects caused by replacing a single element in a periodic array with an irregular scatterer. The parameters used are $a=0.25, k=5.0$, and $\Theta=0.25 \pi$, and a Dirichlet boundary condition is applied on the surface of the regular scatterers (shown as white with a black boundary). In Figure 5.4(a), there is no defect, and the quasi-periodic nature of the field is evident. In Figure 5.4(b), the field is modified using the solutions to the canonical source problems, so that the Neumann boundary condition is now satisfied on the surface of scatterer 0 (shown as black with a white boundary). Contour lines intersecting this scatterer do so at a right angle to the surface tangent. The influence of the defect is more significant in the region above the array, because the field in the periodic case is relatively weak here.

For suitable parameters, irregular scatterers also cause RB waves to be excited. Figure 5.5 shows contour plots of $\left|\Gamma^{+}\right|$for $a=0.25$ with varying $k$ and $\Theta$, with $\mathcal{D}=\{0\}$ and $\mathcal{D}=\{0,1,2,3,4,5\}$. The defects consist of Dirichlet scatterers with radius $a=0.25$. As before, $\left|\Gamma^{-}\right|$can be deduced by symmetry. The pattern of behavior here is quite different from the case of absent scatterers shown in Figure 5.1. The main qualitative difference lies in the dependence of $\left|\Gamma^{+}\right|$upon $\Theta$; there is no longer a second peak at $\Theta \approx 0.8 \pi$. Elsewhere, the excitation is generally stronger than it is in the corresponding cases in Figure 5.1.

5.3. Widely spaced defects. If the defects are spread over a large section of the array, the evaluation of (4.9) by quadrature is no longer straightforward. This is because we must calculate values for $C_{m, n}^{p-q, 0}$ for all $p, q \in \mathcal{D}$, and if $|p-q|$ is large, the integrals are difficult to compute. There are a number of ways to proceed. One possibility is to adopt a mixed strategy, obtaining $\Gamma^{ \pm}$using the MASM, and then solving for the decaying contributions to $C_{m, n}^{p-q, 0}$ using the infinite array subtraction technique discussed in section 5.1. This yields approximate values for all of the unknown coefficients, and is therefore a particularly attractive idea if results for a large number of different defect sets are to be computed. Alternatively, we can form 
(a)

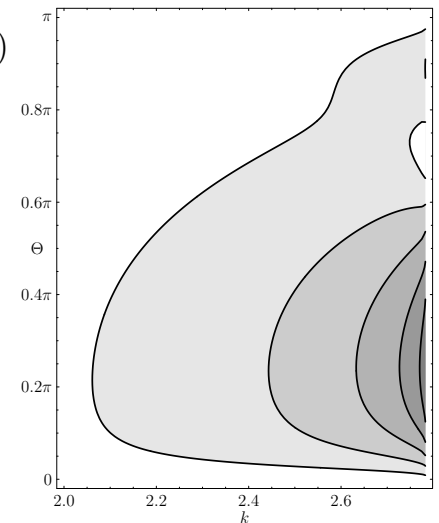

(b)

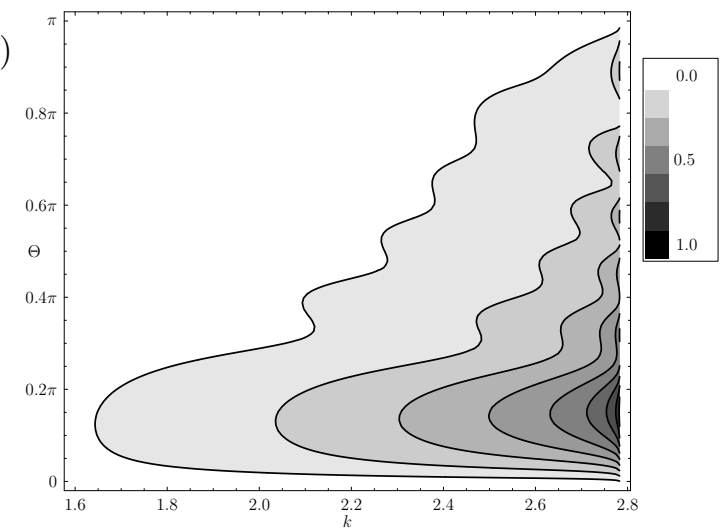

FIG. 5.5. Contour plots showing the amplitude of the right-propagating $R B$ wave, $\left|\Gamma^{+}\right|$, for $a=0.25$ with varying $k$ and $\Theta$; Dirichlet boundary conditions are applied on the surface of scatterers for which $p \in \mathcal{D}$. (a) $\mathcal{D}=\{0\}$. (b) $\mathcal{D}=\{0,1,2,3,4,5\}$.

an approximate interaction theory by neglecting the decaying terms in (4.21) when $p^{3 / 2} \gg 1$. This approximation was used in generating the data for Figure 5.6; it amounts to assuming that significant interactions between widely spaced defects are caused solely by the RB modes and has been shown to work well in practice in the related case of a long, finite array [24].

The presence of such widely spaced defects in an array can lead to "near-trapping" in the intermediate region. This effect was originally reported in [13] in a study of interactions between water waves and long, finite arrays of bottom-mounted circular cylinders. In this physical context, the force in the $x$ direction exerted on cylinder $p$ by the total field (i.e., the integral of the pressure times the component of the outgoing normal to $r_{p}=a$ that is parallel to the array), normalized using the force exerted on an isolated cylinder, is given in [9] as

$$
X_{p}=\left|\frac{1}{2 Z_{1}}\left[A_{1}^{p}(\mathcal{D})-A_{-1}^{p}(\mathcal{D})\right]\right| .
$$

Figure 5.6 shows a contour plot of the horizontal force on an array element that is equidistant between two widely spaced defects, each of which consists of a single absent element. The scatterer radius $a$ is 0.25 , as in [13] and in the majority of cases in [24]. The wavenumber is varied between 2.7 and the cut-off for $\mathrm{RB}$ waves $(k \approx 2.783)$, using 1000 data points, and the angle of incidence is varied between 0 and $\pi / 2$ using 500 data points. Results for $\Theta>\pi / 2$ can be deduced by symmetry. The plot reveals that very large forces occur at certain discrete intervals in $k$ and $\Theta$. The strongest force occurs at a wavenumber that is close to, but not exactly equal to, the cut-off for RB waves. No significant peaks in the force occur for values of $k$ smaller than those shown. The causes of the near-trapping effect are explored in [24] for the case of a finite array; the mechanism here is much the same. Essentially, RB waves generated by one periodicity breaking feature (end or defect) are reflected back by the other. The magnitude of the reflection coefficient increases as $k \rightarrow k_{\max }$. Peaks in the force correspond to situations where the interference is predominantly constructive between $\mathrm{RB}$ modes excited by the interaction of the incident wave with the defects and those generated by reflection. 


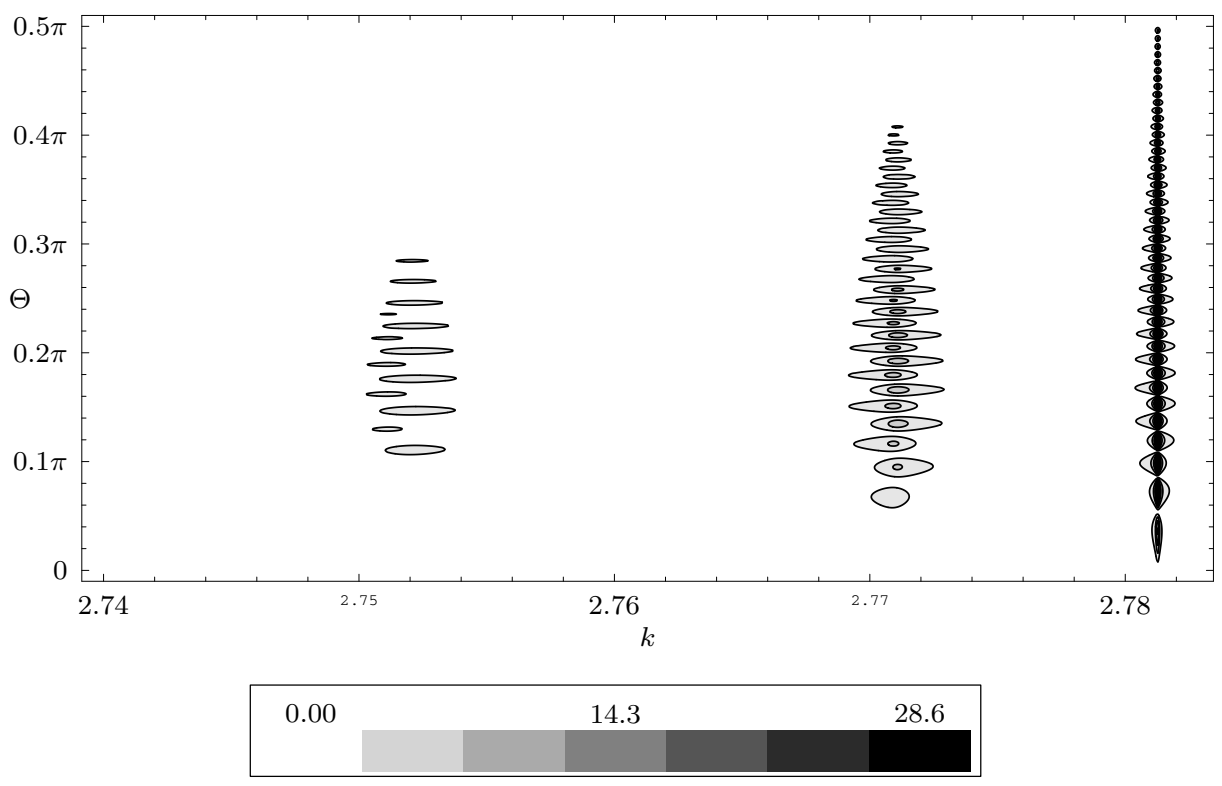

FIG. 5.6. Contour plot showing the force exerted on scatterer 51 , with $a=0.25$ and $\mathcal{D}=\{0,102\}$.

6. Concluding remarks. By reducing the problem of scattering by a defective array to a set of simpler, canonical problems, we have developed an interaction theory for defects in infinite periodic arrays. This is similar in nature to the standard interaction theory for a finite number of bodies. The simplest case is that of an array with one or more absent scatterers. A straightforward extension to the theory that allows irregular scatterers to be considered has also been presented. The MASM is an effective means by which the canonical problems can be solved, and in particular enables important field characteristics such as RB surface wave amplitudes to be efficiently calculated to near machine accuracy. The canonical problems are independent of the defect type and configuration and all aspects of the incident field except the wavenumber, and need not be solved again if these parameters are changed.

Numerical results for various cases have been presented, with particular attention paid to the amplitude with which RB surface waves are excited. The MASM is particularly well-suited to cases in which the defects are localized. For defects that are spread over a larger section of the array, we have shown how other methods such as infinite array subtraction and the large array approximation can be incorporated so as to overcome the difficulties that arise. All of the results that we have presented involve arrays whose elements are circular cylinders. It is not difficult to modify our theory so as to account for other shapes by using transfer matrices. More complicated cases such as fully three-dimensional scattering problems can also be considered, provided that the relevant analogue to the theory of periodic arrays summarized in section 3 is available.

Appendix. Numerical quadrature. The most computationally expensive procedure in applying the interaction theory for defects in arrays is the evaluation of the integrals in (4.16) and (4.9). Quadratures must be performed on a contour whose orientation with respect to the branch points is the same as that shown in Figure 4.1, but in general it is convenient to move the path of integration away from 

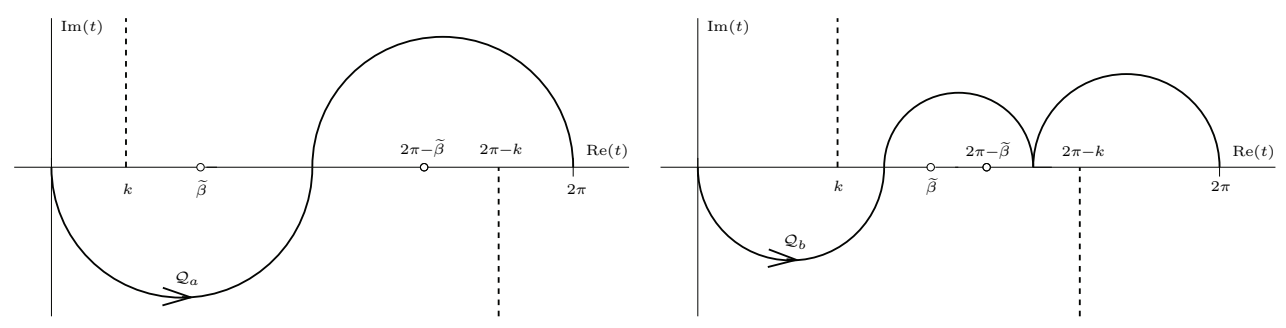

FIG. A.1. Schematic diagrams of the contours $\mathcal{Q}_{a}$ and $\mathcal{Q}_{b}$ used for numerical quadrature.

the various singularities. Since the residues of the functions $f_{m, m}(t)$ and $g_{m, n}(t)$ can be obtained using (4.20), the orientation with respect to the poles need not be maintained. In choosing an appropriate contour, a number of factors must be taken into consideration. These include the possibility of complex poles, contour length, proximity to the known real line singularities, and the behavior of the exponential term $\mathrm{e}^{\mathrm{i} p t}$ that appears in (4.9). Obviously, the extent to which a computer program can automatically adjust the contour to account for these factors has a significant effect on its overall complexity.

The paths of integration used by our numerical codes when RB modes are present are shown in Figure A.1. These are chosen for their relative simplicity, and we do not claim that they are optimal. For most parameter values, the distance between the two poles is at least as great as the distance between a pole and the nearest branch point, i.e., $2(\pi-\widetilde{\beta}) \geq \widetilde{\beta}-k$, and so we use the contour $\mathcal{Q}_{a}$, which consists of two semicircular $\operatorname{arcs}$ of radius $\pi / 2$, centered at $t=\pi / 2$ and $t=3 \pi / 2$. As $k \rightarrow \pi, \widetilde{\beta} \rightarrow \pi$, and so the two poles move close together. If $2(\pi-\widetilde{\beta})<\widetilde{\beta}-k$, then we integrate along $\mathcal{Q}_{b}$, which consists of two semicircular arcs of radius $(\widetilde{\beta}+k) / 4$ centered at $t=(\widetilde{\beta}+k) / 4$ and $t=2 \pi-(\widetilde{\beta}+k) / 4$, and a third arc centered at $t=\pi$ with radius $2 \pi-(\widetilde{\beta}+k)$. A residue contribution from the pole at $t=\widetilde{\beta}$ must be included in this case.

To deal with the possibility of complex poles, we introduce the function $d(t)$ as the determinant of the matrix on the left-hand side of (4.11) (also (4.14)), so that poles of $f_{m}^{n}(t)$ and $g_{m}^{n}(t)$ can occur only at points where $d(t)=0$. We then numerically apply the principle of the argument [17, page 99] to $\log [d(t)]$ in the finite region(s) of the cut plane enclosed by the original path of integration (Figure 4.1) and the new contour (Figure A.1). Aside from $t=\widetilde{\beta}$ and $t=2 \pi-\widetilde{\beta}$, no poles that interfere with the deformations used here have been found. Additional poles were found on the line $t=\pi+\mathrm{i} u, u \in \mathbb{R}$, but only for large values of $|u|$. It should be noted that we have not searched exhaustively across the parameter ranges for $a$ and $k$. A uniform partition of the contours $\mathcal{Q}_{a}$ and $\mathcal{Q}_{b}$ is used by our numerical codes, and the three-point Gaussian formula is applied on each subinterval. In cases involving multiple defects, efficiency can be greatly improved by storing values of $f_{m, n}(t)$ at the partition points used for the largest value of $|p|$ at which the integral in (4.9) must be evaluated and by making repeated use of these.

It is of some interest to compare the accuracy achieved by the MASM with that of the filtering technique [11], which can also be used to solve the canonical problems. The filtering technique requires the truncation and inversion of linear systems involving slowly convergent infinite spatial sums. We should therefore expect the MASM to achieve a superior degree of both accuracy and performance. An ideal parameter 
TABLE A.1

Convergence in $\left|c_{0}\right|$ and performance in computing $\left|c_{n}\right|$ for $a=0.25$ and $k=2.5$.

\begin{tabular}{rccr||rrrr}
\hline \multicolumn{4}{c||}{ Modified array scanning } & \multicolumn{4}{c}{ Filtering } \\
\hline NSI & $\left|c_{0}\right|$ & \% change & time (s) & SPT & $\left|c_{0}\right|$ & \% change & time (s) \\
99 & 0.1361053213 & & 4.24 & 70 & 0.1360833934 & & 4.36 \\
149 & 0.1361053214 & 0.0000000039 & 6.40 & 90 & 0.1360096190 & 0.0542126301 & 8.55 \\
199 & 0.1361053214 & 0.0000000003 & 8.57 & 110 & 0.1361554350 & 0.1072100718 & 14.33 \\
249 & 0.1361053214 & 0.0000000000 & 10.72 & 130 & 0.1361523637 & 0.0022557485 & 21.68 \\
299 & 0.1361053214 & 0.0000000000 & 12.90 & 150 & 0.1360562815 & 0.0705696206 & 31.55 \\
\hline
\end{tabular}

for comparison is the quantity $\left|c_{n}\right|$, i.e., the amplitude of the RB wave that is excited by a source of order $n$ replacing the scatterer centered at the origin. Table A.1 shows typical performance and accuracy figures that can be achieved by the two methods. The parameters used are $a=0.25$ and $k=1$, which lead to $\widetilde{\beta} \approx 2.586$, and the computations are performed using Fortran 2003 on a machine with a $2.5 \mathrm{GHz}$ processor. Note that the times given are those required for the simultaneous computation of $c_{n}$ for all $n$ up to the order truncation. The abbreviation NSI stands for the number of subintervals into which the contour is divided. The value for the spatial index $p$ at which the linear system used in the filtering method is truncated is denoted by SPT. The dependence of computation time upon NSI is clearly linear, whereas increasing SPT leads to a significant decrease in performance. The results obtained by the two methods are in agreement up to the degree of accuracy that can be expected of the filtering method [11]; this requires the inversion of a large linear system of equations and is susceptible to round-off errors. It is clear that the MASM yields far greater accuracy, and is also much more efficient.

\section{REFERENCES}

[1] H. Ammari and F. Santosa, Guided waves in a photonic bandgap structure with a line defect, SIAM J. Appl. Math., 64 (2004), pp. 2018-2033.

[2] A.-S. Bonnet-Bendhia And F. Starling, Guided waves by electromagnetic gratings and nonuniqueness examples for the diffraction problem, Math. Methods Appl. Sci., 17 (1994), pp. 305-338.

[3] F. Capolino, D. R. Jackson, And D. R. Wilton, Fundamental properties of the field at the interface between air and a periodic artificial material excited by a line source, IEEE Trans. Antennas and Propagation, 53 (2005), pp. 91-99.

[4] F. Capolino, D. R. Jackson, and D. R. Wilton, Mode excitation from sources in twodimensional EBG waveguides using the array scanning method, IEEE Microwave and Wireless Components Letters, 15 (2005), pp. 49-51.

[5] K. B. Dossou, L. C. Botten, S. Wilcox, R. C. McPhedran, C. M. de Sterke, N. A. Nicorovici, AND A. A. AsATrYAn, Exact modelling of generalised defect modes in photonic crystal structures, Phys. B, 394 (2007), pp. 330-334.

[6] D. V. Evans And R. Porter, Trapping and near-trapping by arrays of cylinders in waves, J. Engrg. Math., 35 (1999), pp. 149-179.

[7] A. Khelif, A. Choujaa, B. Duafari-Rouhani, M. Wilm, S. Ballandras, and V. Laude, Trapping and guiding of acoustic waves by defect modes in a full-band-gap ultrasonic crystal, Phys. Rev. B, 68 (2003), 214301.

[8] C. M. Linton, Schlömilch series that arise in diffraction theory and their efficient computation, J. Phys. A, 39 (2006), pp. 3325-3339.

[9] C. M. Linton And D. V. Evans, The interaction of waves with arrays of vertical circular cylinders, J. Fluid Mech., 215 (1990), pp. 549-569.

[10] C. M. Linton And M. McIver, The existence of Rayleigh-Bloch surface waves, J. Fluid Mech., 470 (2002), pp. 85-90.

[11] C. M. Linton, R. Porter, And I. Thompson, Scattering by a semi-infinite periodic array and the excitation of surface waves, SIAM J. Appl. Math., 67 (2007), pp. 1233-1258.

Copyright (c) by SIAM. Unauthorized reproduction of this article is prohibited. 
[12] C. M. Linton And I. Thompson, Resonant effects in scattering by periodic arrays, Wave Motion, 44 (2007), pp. 167-175.

[13] H. D. Maniar and J. N. Newman, Wave diffraction by a long array of cylinders, J. Fluid Mech., 339 (1997), pp. 309-330.

[14] P. A. Martin, Multiple Scattering. Interaction of Time-Harmonic Waves with $N$ Obstacles, Cambridge University Press, Cambridge, UK, 2006.

[15] P. McIver, C. M. Linton, And M. McIver, Construction of trapped modes for wave guides and diffraction gratings, R. Soc. Lond. Proc. Ser. A Math. Phys. Eng. Sci., 454 (1998), pp. 2593-2616.

[16] B. A. Munk And G. A. Burrell, Plane-wave expansion for arrays of arbitrarily oriented piecewise linear elements and its application in determining the impedance of a single linear antenna in a lossy half-space, IEEE Trans. Antennas and Propagation, 27 (1979), pp. 331-343.

[17] A. D. Osbonne, Complex Variables and Their Applications, Int. Math. Ser., Addison Wesley Longman Limited, Harlow, UK, 1999.

[18] R. PORTER AND D. V. Evans, Rayleigh-Bloch surface waves along periodic gratings and their connection with trapped modes in waveguides, J. Fluid Mech., 386 (1999), pp. 233-258.

[19] M. M. Sigalas, Elastic wave band gaps and defect states in two-dimensional composites, J. Acoust. Soc. Amer., 101 (1997), pp. 1256-1261.

[20] I. Stakgold, Green's Functions and Boundary Value Problems, 2nd ed., Wiley, New York, 1998.

[21] S. V. Sukhinin, The whispering surface effect, J. Appl. Math. Mech., 63 (1999), pp. 863-876.

[22] I. Thompson and C. M. Linton, An embedding method for scattering by defective arrays, in Proceedings of Waves 2007, Reading, UK, 2007, pp. 203-205.

[23] I. Thompson And C. M. Linton, On the excitation of a closely spaced array by a line source, IMA J. Appl. Math., 72 (2007), pp. 476-497.

[24] I. Thompson, C. M. Linton, And R. Porter, A new approximation method for scattering by long finite arrays, Quart. J. Mech. Appl. Math., published online March 27, 2008; doi:10.1093/qjmam/hbn006.

[25] V. Twersky, Elementary function representation of Schlömilch series, Arch. Rational Mech. Anal., 8 (1961), pp. 323-332.

[26] S. Wilcox, L. C. Botten, R. C. McPhedran, C. G. Poulton, and C. Martijn de Sterke, Modeling of defect modes in photonic crystals using the fictitious source superposition method, Phys. Rev. E, 71 (2005), 056606.

[27] C. P. Wu And V. Galindo, Properties of a phased array of rectangular waveguides with thin walls, IEEE Trans. Antennas and Propagation, 14 (1966), pp. 163-173.

[28] F. Wu, Z. Liu, AND Y. Liu, Splitting and tuning characteristics of the point defect modes in two-dimensional phononic crystals, Phys. Rev. E, 69 (2004), 066609.

Copyright (c) by SIAM. Unauthorized reproduction of this article is prohibited. 